\title{
Fuzzy Entropy for Pythagorean Fuzzy Sets with Application to Multicriterion Decision Making
}

\author{
Miin-Shen Yang $\mathbb{D}^{1}$ and Zahid Hussain ${ }^{1,2}$ \\ ${ }^{1}$ Department of Applied Mathematics, Chung Yuan Christian University, Chung-Li 32023, Taiwan \\ ${ }^{2}$ Department of Mathematics, Karakoram International University, Gilgit-Baltistan, Pakistan \\ Correspondence should be addressed to Miin-Shen Yang; msyang@math.cycu.edu.tw
}

Received 17 June 2018; Revised 1 October 2018; Accepted 16 October 2018; Published 1 November 2018

Academic Editor: Diego R. Amancio

Copyright (c) 2018 Miin-Shen Yang and Zahid Hussain. This is an open access article distributed under the Creative Commons Attribution License, which permits unrestricted use, distribution, and reproduction in any medium, provided the original work is properly cited.

\begin{abstract}
The concept of Pythagorean fuzzy sets (PFSs) was initially developed by Yager in 2013, which provides a novel way to model uncertainty and vagueness with high precision and accuracy compared to intuitionistic fuzzy sets (IFSs). The concept was concretely designed to represent uncertainty and vagueness in mathematical way and to furnish a formalized tool for tackling imprecision to real problems. In the present paper, we have used both probabilistic and nonprobabilistic types to calculate fuzzy entropy of PFSs. Firstly, a probabilistic-type entropy measure for PFSs is proposed and then axiomatic definitions and properties are established. Secondly, we utilize a nonprobabilistic-type with distances to construct new entropy measures for PFSs. Then a min-max operation to calculate entropy measures for PFSs is suggested. Some examples are also used to demonstrate suitability and reliability of the proposed methods, especially for choosing the best one/ones in structured linguistic variables. Furthermore, a new method based on the chosen entropies is presented for Pythagorean fuzzy multicriterion decision making to compute criteria weights with ranking of alternatives. A comparison analysis with the most recent and relevant Pythagorean fuzzy entropy is conducted to reveal the advantages of our developed methods. Finally, this method is applied for ranking China-Pakistan Economic Corridor (CPEC) projects. These examples with applications demonstrate practical effectiveness of the proposed entropy measures.
\end{abstract}

\section{Introduction}

The concept of fuzzy sets was first proposed by Zadeh [1] in 1965 . With a widely spread use in various fields, fuzzy sets not only provide broad opportunity to measure uncertainties in more powerful and logical way, but also give us a meaningful way to represent vague concepts in natural language. It is known that most systems based on 'crisp set theory' or 'twovalued logics' are somehow difficult for handling imprecise and vague information. In this sense, fuzzy sets can be used to provide better solutions for more real world problems. Moreover, to treat more imprecise and vague information in daily life, various extensions of fuzzy sets are suggested by researchers, such as interval-valued fuzzy set [2], type-2 fuzzy sets [3], fuzzy multiset [4], intuitionistic fuzzy sets [5], hesitant fuzzy sets $[6,7]$, and Pythagorean fuzzy sets $[8,9]$.

Since fuzzy sets were based on membership values or degrees between 0 and 1 , in real life setting it may not be always true that nonmembership degree is equal to (1membership). Therefore, to get more purposeful reliability and applicability, Atanassov [5] generalized the concept of 'fuzzy set theory' and proposed Intuitionistic fuzzy sets (IFSs) which include both membership degree and nonmembership degree and degree of nondeterminacy or uncertainty where degree of uncertainty $=(1-$ (degree of membership + nonmembership degree)). In IFSs, the pair of membership grades is denoted by $(\mu, \nu)$ satisfying the condition of $\mu+\nu \leq 1$. Recently, Yager and Abbasov [8] and Yager [9] extended the condition $\mu+\nu \leq 1$ to $\mu^{2}+\nu^{2} \leq 1$ and then introduced a class of Pythagorean fuzzy sets (PFSs) whose membership values are ordered pairs $(\mu, \nu)$ that fulfills the required condition of $\mu^{2}+\nu^{2} \leq 1$ with different aggregation operations and applications in multicriterion decision making. According to Yager and Abbasov [8] and Yager [9], the space of all intuitionistic membership values (IMVs) is also Pythagorean 
membership values (PMVs), but PMVs are not necessary to be IMVs. For instance, for the situation when the numbers $\mu=\sqrt{3} / 2$ and $\nu=1 / 2$, we can use PFSs, but IFSs cannot be used since $\mu+\nu>1$, but $\mu^{2}+\nu^{2} \leq 1$. PFSs are wider than IFSs so that they can tackle more daily life problems under imprecision and uncertainty cases.

More researchers are actively engaged in the development of PFSs properties. For example, Yager [10] gave Pythagorean membership grades in multicriterion decision making. Extensions of technique for order preference by similarity to an ideal solution (TOPSIS) to multiple criteria decision making with Pythagorean and hesitant fuzzy sets were proposed by Zhang and Xu [11]. Zhang [12] considered a novel approach based on similarity measure for Pythagorean fuzzy multicriteria group decision making. Pythagorean fuzzy TODIM approach to multicriterion decision making was given by Ren et al. [13]. Pythagorean fuzzy Choquet integral based MABAC method for multiple attribute group decision making was developed by Peng and Yang [14]. Zhang [15] gave a hierarchical QUALIFLEX approach. Peng et al. [16] investigated Pythagorean fuzzy information measures. Zhang et al. [17] proposed generalized Pythagorean fuzzy Bonferroni mean aggregation operators. Liang and Xu [18] extended TOPSIS to hesitant Pythagorean fuzzy sets. PérezDomínguez et al. [19] gave MOORA under Pythagorean fuzzy sets. Recently, Pythagorean fuzzy LINMAP method based on the entropy for railway project investment decision making was proposed by Xue et al. [20]. Zhang and Meng [21] proposed an approach to interval-valued hesitant fuzzy multiattribute group decision making based on the generalized Shapley-Choquet integral. Pythagorean fuzzy $(\mathrm{R}, \mathrm{S})$ - norm information measure for multicriteria decision making problem was presented by Guleria and Bajaj [22]. Furthermore, Yang and Hussain [23] proposed distance and similarity measures of hesitant fuzzy sets based on Hausdorff metric with applications to multicriteria decision making and clustering. Hussain and Yang [24] gave entropy for hesitant fuzzy sets based on Hausdorff metric with construction of hesitant fuzzy TOPSIS.

The entropy of fuzzy sets is a measure of fuzziness between fuzzy sets. De Luca and Termini [25] first introduced the axiom construction for entropy of fuzzy sets with reference to Shannon's probability entropy. Yager [26] defined fuzziness measures of fuzzy sets in terms of a lack of distinction between the fuzzy set and its negation based on Lp norm. Kosko [27] provided a measure of fuzziness between fuzzy sets using a ratio of distance between the fuzzy set and its nearest set to the distance between the fuzzy set and its farthest set. Liu [28] gave some axiom definitions of entropy and also defined a $\sigma$-entropy. Pal and Pal [29] proposed exponential entropies. While Fan and $\mathrm{Ma}$ [30] gave some new fuzzy entropy formulas. Some extended entropy measures for IFS were proposed by Burillo and Bustince [31], Szmidt and Kacprzyk [32], Szmidt and Baldwin [33], and Hung and Yang [34].

In this paper, we propose new entropies of PFS based on probability-type, distance, Pythagorean index, and min-max operation. We also extend the concept to $\sigma$-entropy and then apply it to multicriteria decision making. This paper is organized as follows. In Section 2, we review some definitions of IFSs and PFSs. In Section 3, we propose several new entropies of PFSs and then construct an axiomatic definition of entropy for PFSs. Based on the definition of entropy for PFSs, we find that the proposed nonprobabilistic entropies of PFSs are $\sigma$-entropy. In Section 4, we exhibit some examples for comparisons and also use structured linguistic variables to validate our proposed methods. In Section 5, we construct a new Pythagorean fuzzy TOPSIS based on the proposed entropy measures. A comparison analysis of the proposed Pythagorean fuzzy TOPSIS with the recently developed entropy of PFS [20] is shown. We then apply the proposed method to multicriterion decision making for ranking ChinaPakistan Economic Corridor projects. Finally, we state our conclusion in Section 6.

\section{Intuitionistic and Pythagorean Fuzzy Sets}

In this section, we give a brief review for intuitionistic fuzzy sets (IFSs) and Pythagorean fuzzy sets (PFSs).

Definition 1. An intuitionistic fuzzy set (IFS) $\widetilde{M}$ in $X$ is defined by Atanassov [5] with the following form:

$$
\widetilde{M}=\left\{\left(x, \mu_{\widetilde{M}}(x), \nu_{\widetilde{M}}(x)\right): x \in X\right\}
$$

where $0 \leq \mu_{\widetilde{M}}(x)+\nu_{\widetilde{M}}(x) \leq 1, \forall x \in X$, and the functions $\mu_{\widetilde{M}}(x): X \longrightarrow[0,1]$ denotes the degree of membership of $x$ in $\widetilde{M}$ and $\nu_{\widetilde{M}}(x): X \longrightarrow[0,1]$ denotes the degree of nonmembership of $x$ in $\widetilde{M}$. The degree of uncertainty (or intuitionistic index, or indeterminacy) of $x$ to $\widetilde{M}$ is represented by $\pi_{\widetilde{M}}(x)=1-\left(\mu_{\widetilde{M}}(x)+\nu_{\widetilde{M}}(x)\right)$.

For modeling daily life problems carrying imprecision, uncertainty, and vagueness more precisely and with high accuracy than IFSs, Yager $[9,10]$ presented Pythagorean fuzzy sets (PFSs), where PFSs are the generalizations of IFSs. Yager $[9,10]$ also validated that IFSs are contained in PFSs. The concept of Pythagorean fuzzy set was originally developed by Yager $[8,9]$, but the general mathematical form of Pythagorean fuzzy set was developed by Zhang and $\mathrm{Xu}$ [11].

Definition 2 (Zhang and $\mathrm{Xu}$ [11]). A Pythagorean fuzzy set (PFS) $\widetilde{P}$ in $X$ proposed by Yager $[8,9]$ is mathematically formed as

$$
\widetilde{P}=\left\{\left\langle x, \mu_{\widetilde{P}}(x), \nu_{\widetilde{P}}(x)\right\rangle: x \in X\right\}
$$

where the functions $\mu_{\widetilde{P}}(x): X \longrightarrow[0,1]$ represent the degree of membership of $x$ in $\widetilde{P}$ and $\nu_{\widetilde{P}}(x): X \longrightarrow[0,1]$ represent the degree of nonmembership of $x$ in $\widetilde{P}$. For every $x \in X$, the following condition should be satisfied:

$$
0 \leq \mu_{\widetilde{P}}^{2}(x)+\nu_{\widetilde{P}}^{2}(x) \leq 1 .
$$


Definition 3 (Zhang and Xu [11]). For any PFS $\widetilde{P}$ in $X$, the value $\pi_{\widetilde{P}}(x)$ is called Pythagorean index of the element $x$ in $\widetilde{P}$ with

$$
\begin{aligned}
\pi_{\widetilde{P}}(x) & =\sqrt{1-\left\{\mu_{\widetilde{P}}^{2}(x)+v_{\widetilde{P}}^{2}(x)\right\}} \\
\text { or } \pi_{\widetilde{P}}^{2}(x) & =1-\mu_{\widetilde{P}}^{2}(x)-v_{\widetilde{P}}^{2}(x) .
\end{aligned}
$$

In general, $\pi_{\widetilde{P}}(x)$ is also called hesitancy (or indeterminacy) degree of the element $x$ in $\widetilde{P}$. It is obvious that $0 \leq$ $\pi_{\widetilde{P}}^{2}(x) \leq 1, \forall x \in X$. It is worthy to note, for a PFS $\widetilde{P}$, if $\mu_{\widetilde{P}}^{2}(x)=0$ then $\nu_{\widetilde{P}}^{2}(x)+\pi_{\widetilde{P}}^{2}(x)=1$, and if $\mu_{\widetilde{P}}^{2}(x)=1$ then $\nu_{\widetilde{P}}^{2}(x)=0$ and $\pi_{\widetilde{P}}^{2}(x)=0$. Similarly, if $\nu_{\widetilde{P}}^{2}(x)=0$ then $\mu_{\widetilde{P}}^{2}(x)+\pi_{\widetilde{P}}^{2}(x)=1$. If $\nu_{\widetilde{P}}^{2}(x)=1$ then $\mu_{\widetilde{P}}^{2}(x)=0$ and $\pi_{\widetilde{P}}^{2}(x)=0$. If $\pi_{\widetilde{P}}^{2}(x)=0$ then $\mu_{\widetilde{P}}^{2}(x)+\nu_{\widetilde{P}}^{2}(x)=1$. If $\pi_{\widetilde{P}}^{2}(x)=1$ then $\mu_{\widetilde{P}}^{2}(x)=\nu_{\widetilde{P}}^{2}(x)=0$. For convenience, Zhang and Xu [11] denoted the pair $\left(\mu_{\tilde{P}}(x), \nu_{\widetilde{P}}(x)\right)$ as Pythagorean fuzzy number (PFN), which is represented by $\widetilde{p}=\left(\mu_{\tilde{p}}, \nu_{\tilde{p}}\right)$.

Since PFSs are a generalized form of IFSs, we give the following definition for PFSs.

Definition 4. Let $\widetilde{P}$ be a PFS in $X . \widetilde{P}$ is called a completely Pythagorean if $\mu_{\widetilde{P}}^{2}(x)=v_{\widetilde{P}}^{2}(x)=0, \forall x \in X$.

Peng et al. [16] suggested various mathematical operations for PFSs as follows:

Definition 5 (Peng et al. [16]). If $\widetilde{P}$ and $\widetilde{Q}$ are two PFSs in $X$, then

(i) $\widetilde{P} \leq \widetilde{Q}$ if and only if $\forall x \in X, \mu_{\widetilde{P}}^{2}(x) \leq \mu_{\widetilde{Q}}^{2}(x)$ and $v_{\widetilde{P}}^{2}(x) \geq v_{\widetilde{Q}}^{2}(x)$

(ii) $\widetilde{P}=\widetilde{Q}$ if and only if $\forall x \in X, \mu_{\widetilde{P}}^{2}(x)=\mu_{\widetilde{Q}}^{2}(x)$ and $v_{\widetilde{P}}^{2}(x)=v_{\widetilde{Q}}^{2}(x)$

(iii) $\widetilde{P} \cup \widetilde{Q}=\left\{\left\langle x, \max \left(\mu_{\widetilde{P}}^{2}(x), \mu_{\widetilde{Q}}^{2}(x)\right), \min \left(\nu_{\widetilde{P}}^{2}(x), v_{\widetilde{Q}}^{2}(x)\right)\right\rangle\right.$ : $x \in X\}$;

(iv) $\widetilde{P} \cap \widetilde{Q}=\left\{\left\langle x, \min \left(\mu_{\widetilde{P}}^{2}(x), \mu_{\widetilde{Q}}^{2}(x)\right), \max \left(v_{\widetilde{P}}^{2}(x), v_{\widetilde{Q}}^{2}(x)\right)\right\rangle\right.$ : $x \in X\}$;

(v) $\widetilde{P}^{c}=\left\{\left\langle x, v_{\widetilde{P}}^{2}(x), \mu_{\widetilde{Q}}^{2}(x)\right\rangle: x \in X\right\}$.

We next define more operations of PFS in $X$, especially about hedges of "very", "highly", "more or less", "concentration", "dilation", and other terms that are needed to represent linguistic variables. We first define the $n$ power (or exponent) of PFS as follows.

Definition 6. Let $\widetilde{P}=\left\{\left\langle x, \mu_{\widetilde{P}}(x), \nu_{\widetilde{P}}(x)\right\rangle: x \in X\right\}$ be a PFS in $X$. For any positive real number $n$, the $n$ power (or exponent) of the PFS $\widetilde{P}$, denoted by $\widetilde{P}^{n}$, is defined as

$$
\widetilde{P}^{n}=\left\{\left\langle x,\left(\mu_{\widetilde{P}}(x)\right)^{n}, \sqrt{1-\left(1-v_{\widetilde{P}}^{2}(x)\right)^{n}}\right\rangle: x \in X\right\} .
$$

It can be easily verified that, for any positive real number $n$, $0 \leq\left[\mu_{\widetilde{P}}(x)\right]^{n}+\left[\sqrt{1-\left(1-v_{\widetilde{P}}^{2}(x)\right)^{n}}\right] \leq 1, \forall x \in X$.
By using Definition 6, the concentration and dilation of a PFS $\widetilde{P}$ can be defined as follows.

Definition 7. The concentration $\operatorname{CON}(\widetilde{P})$ of a PFS $\widetilde{P}$ in $X$ is defined as

$$
\operatorname{CON}(\widetilde{P})=\left\{\left\langle x, \mu_{\mathrm{CON}(\widetilde{P})}(x), \nu_{\mathrm{CON}(\widetilde{P})}(x)\right\rangle: x \in X\right\}
$$

where $\mu_{\mathrm{CON}(\widetilde{P})}(x)=\left[\mu_{\widetilde{P}}(x)\right]^{2}$ and $v_{\mathrm{CON}(\widetilde{P})}(x)=$ $\sqrt{1-\left[1-v_{\tilde{P}}^{2}(x)\right]^{2}}$.

Definition 8. The dilation $\operatorname{DIL}(\widetilde{P})$ of a PFS $\widetilde{P}$ in $X$ is defined as

$$
\operatorname{DIL}(\widetilde{P})=\left\{\left\langle x, \mu_{D I L(\widetilde{P})}(x), v_{D I L(\widetilde{P})}(x)\right\rangle: x \in X\right\}
$$

where $\mu_{D I L(\widetilde{P})}(x)=\left[\mu_{\widetilde{P}}(x)\right]^{1 / 2}$ and $v_{D I L(\widetilde{P})}(x)=$ $\sqrt{1-\left[1-v_{\widetilde{P}}^{2}(x)\right]^{1 / 2}}$.

In next section, we construct new entropy measures for PFSs based on probability-type, entropy induced by distance, Pythagorean index, and max-min operation. We also give an axiomatic definition of entropy for PFSs.

\section{New Fuzzy Entropies for Pythagorean Fuzzy Sets}

We first provide a definition of entropy for PFSs. De Luca and Termini [25] gave the axiomatic definition of entropy measure of fuzzy sets. Later on, Szmidt and Kacprzyk [32] extended it to entropy of IFS. Since PFSs developed by Yager $[8,9]$ are generalized forms of IFSs, we use similar notions as IFSs to give a definition of entropy for PFSs. Assume that $P F S(X)$ represents the set of all PFSs in $X$.

Definition 9. A real function $E: \operatorname{PFS}(X) \longrightarrow[0,1]$ is called an entropy on $\operatorname{PFS}(X)$ if E satisfies the following axioms:

(A0) (Nonnegativity) $0 \leq E(\widetilde{P}) \leq 1$;

(A1) (Minimality) $E(\widetilde{P})=0$, iff $\widetilde{P}$ is a crisp set;

(A2) (Maximality) $E(\widetilde{P})=1$, iff $\mu_{\widetilde{P}}(x)=\nu_{\widetilde{P}}(x), \forall x \in X$;

(A3) (Resolution) $E(\widetilde{P}) \leq E(\widetilde{Q})$, if $\widetilde{P}$ is crisper than $\widetilde{Q}$, i.e., $\forall x \in X$,

$$
\begin{aligned}
& \mu_{\widetilde{P}}(x) \leq \mu_{\widetilde{Q}}(x) \text { and } \nu_{\widetilde{P}}(x) \geq \nu_{\widetilde{Q}}(x) \text { for } \mu_{\widetilde{Q}}(x) \leq \\
& \nu_{\widetilde{Q}}(x) \text { or } \\
& \mu_{\widetilde{P}}(x) \geq \mu_{\widetilde{Q}}(x) \text { and } \nu_{\widetilde{P}}(x) \leq \nu_{\widetilde{Q}}(x) \text { for } \mu_{\widetilde{Q}}(x) \geq \\
& \nu_{\widetilde{Q}}(x) ;
\end{aligned}
$$

(A4) (Symmetric) $E(\widetilde{P})=E\left(\widetilde{P}^{c}\right)$, where $\widetilde{P}^{c}$ is the complement of $\widetilde{P}$;

For probabilistic-type entropy, we need to omit the axiom (A0). On the other hand, because we take the three-parameter $\mu_{\widetilde{P}}^{2}, \nu_{\widetilde{P}}^{2}$, and $\pi_{\widetilde{P}}^{2}$ as a probability mass function $p=\left\{\mu_{\widetilde{P}}^{2}, \nu_{\widetilde{P}}^{2}, \pi_{\widetilde{P}}^{2}\right\}$, the probabilistic-type entropy $E(\widetilde{P})$ should attain a unique 
maximum at $\mu_{\widetilde{P}}(x)=\nu_{\widetilde{P}}(x)=\pi_{\widetilde{P}}(x)=1 / \sqrt{3}, \forall x \in$ $X$. Therefore, for probabilistic-type entropy, we replace the axiom $(A 2)$ with $\left(A 2^{\prime}\right)$ and the axiom $(A 3)$ with $\left(A 3^{\prime}\right)$ as follows:

$\left(A 2^{\prime}\right)$ (Maximality) $E(\widetilde{P})$ attains a unique maximum at $\mu_{\widetilde{P}}(x)=\nu_{\widetilde{P}}(x)=\pi_{\widetilde{P}}(x)=1 / \sqrt{3}, \forall x \in X$.

$\left(A 3^{\prime}\right)$ (Resolution) $E(\widetilde{P}) \leq E(\widetilde{Q})$, if $\widetilde{P}$ is crisper than $\widetilde{Q}$, i.e.," $\forall x \in X, \mu_{\widetilde{P}}(x) \leq \mu_{\widetilde{Q}}(x)$ and $\nu_{\widetilde{P}}(x) \leq \nu_{\widetilde{Q}}(x)$

for $\max \left(\mu_{\widetilde{\mathrm{Q}}}(x), \nu_{\widetilde{\mathrm{Q}}}(x)\right) \leq 1 / \sqrt{3}$ and $\mu_{\widetilde{P}}(x) \geq \mu_{\widetilde{\mathrm{Q}}}(x)$, $\nu_{\widetilde{P}}(x) \geq v_{\widetilde{Q}}(x)$ for $\min \left(\mu_{\widetilde{Q}}(x), \nu_{\widetilde{Q}}(x)\right) \geq 1 / \sqrt{3}$.

In addition to the five axioms (A0) (A4) in Definition 9, if we add the following axiom (A5), E is called $\sigma$ - entropy:

(A5) (Valuation) $E(\widetilde{P})+E(\widetilde{Q})=E(\widetilde{P} \cup \widetilde{Q})+E(\widetilde{P} \cap \widetilde{Q})$, if $\forall x \in X$

$$
\begin{aligned}
& \mu_{\widetilde{P}}(x) \leq \mu_{\widetilde{Q}}(x) \text { and } \nu_{\widetilde{P}}(x) \geq \nu_{\widetilde{Q}}(x) \text { for } \mu_{\widetilde{Q}}(x) \leq \\
& \nu_{\widetilde{Q}}(x) \text { or } \\
& \mu_{\widetilde{P}}(x) \geq \mu_{\widetilde{Q}}(x) \text { and } \nu_{\widetilde{P}}(x) \leq \nu_{\widetilde{Q}}(x) \text { for } \mu_{\widetilde{Q}}(x) \geq \\
& \nu_{\widetilde{Q}}(x) .
\end{aligned}
$$

We present a property for the axiom $\left(A 3^{\prime}\right)$ when $\widetilde{P}$ is crisper than $\widetilde{Q}$.

Property 10. If $\widetilde{P}$ is crisper than $\widetilde{Q}$ in the axiom $\left(A 3^{\prime}\right)$, then we have the following inequality:

$$
\begin{aligned}
& \left(\mu_{\widetilde{P}}\left(x_{i}\right)-\frac{1}{\sqrt{3}}\right)^{2}+\left(\nu_{\widetilde{P}}\left(x_{i}\right)-\frac{1}{\sqrt{3}}\right)^{2} \\
& \quad+\left(\pi_{\widetilde{P}}\left(x_{i}\right)-\frac{1}{\sqrt{3}}\right)^{2} \\
& \geq\left(\mu_{\widetilde{Q}}\left(x_{i}\right)-\frac{1}{\sqrt{3}}\right)^{2}+\left(\nu_{\widetilde{Q}}\left(x_{k}\right)-\frac{1}{\sqrt{3}}\right)^{2} \\
& \quad+\left(\nu_{\widetilde{Q}}\left(x_{k}\right)-\frac{1}{\sqrt{3}}\right)^{2}, \quad \forall x_{i}
\end{aligned}
$$

$$
e_{H C}^{\gamma}(\widetilde{P})= \begin{cases}\frac{1}{n} \sum_{i=1}^{n} \frac{1}{\gamma-1}\left[1-\left(\left(\mu_{\widetilde{P}}^{2}\left(x_{i}\right)\right)^{\gamma}+\left(\nu_{\widetilde{P}}^{2}\left(x_{i}\right)\right)^{\gamma}+\left(\pi_{\widetilde{P}}^{2}\left(x_{i}\right)\right)^{\gamma}\right)\right], & \gamma \neq 1(\gamma>0) \\ \frac{1}{n} \sum_{i=1}^{n}-\left(\mu_{\widetilde{P}}^{2}\left(x_{i}\right) \log \mu_{\widetilde{P}}^{2}\left(x_{i}\right)+v_{\widetilde{P}}^{2}\left(x_{i}\right) \log \nu_{\widetilde{P}}^{2}\left(x_{i}\right)+\pi_{\widetilde{P}}^{2}\left(x_{i}\right) \log \pi_{\widetilde{P}}^{2}\left(x_{i}\right)\right), & \gamma=1 .\end{cases}
$$

Proof. If $\widetilde{P}$ is crisper than $\widetilde{Q}$, then $\forall x_{i}, \mu_{\widetilde{P}}(x) \leq \mu_{\widetilde{Q}}(x)$ and $\nu_{\widetilde{P}}(x) \leq \nu_{\widetilde{Q}}(x)$ for $\max \left(\mu_{\widetilde{Q}}(x), \nu_{\widetilde{Q}}(x)\right) \leq 1 / \sqrt{3}$. Therefore, we have that $\mu_{\widetilde{P}}(x)-1 / \sqrt{3} \leq \mu_{\widetilde{Q}}(x)-1 / \sqrt{3} \leq 0$ and $\nu_{\widetilde{P}}(x)-1 / \sqrt{3} \leq \nu_{\widetilde{Q}}(x)-1 / \sqrt{3} \leq 0$ and so $1-\mu_{\widetilde{P}}^{2}(x)-v_{\widetilde{P}}^{2}(x) \geq$ $1-\mu_{\widetilde{\mathrm{Q}}}^{2}(x)-v_{\widetilde{\mathrm{Q}}}^{2}(x) \geq 1 / 3$, i.e., $\pi_{\widetilde{P}}^{2}(x) \geq \pi_{\widetilde{\mathrm{Q}}}^{2}(x) \geq 1 / 3$ and $\pi_{\widetilde{P}}(x)-1 / \sqrt{3} \geq \pi_{\widetilde{Q}}(x)-1 / \sqrt{3} \geq 0$. Thus, we have $\left(\mu_{\widetilde{P}}(x)-1 / \sqrt{3}\right)^{2} \geq\left(\mu_{\widetilde{Q}}(x)-1 / \sqrt{3}\right)^{2},\left(\nu_{\widetilde{P}}(x)-1 / \sqrt{3}\right)^{2} \geq$ $\left(\nu_{\widetilde{Q}}(x)-1 / \sqrt{3}\right)^{2}$, and $\left(\pi_{\widetilde{P}}(x)-1 / \sqrt{3}\right)^{2} \geq\left(\pi_{\widetilde{Q}}(x)-1 / \sqrt{3}\right)^{2}$. This induces the inequality. Similarly, the part of $\mu_{\widetilde{P}}(x) \geq$ $\mu_{\widetilde{\mathrm{Q}}}(x)$ and $\nu_{\widetilde{P}}(x) \geq \nu_{\widetilde{\mathrm{Q}}}(x)$ for $\min \left(\mu_{\widetilde{\mathrm{Q}}}(x), \nu_{\widetilde{\mathrm{Q}}}(x)\right) \geq 1 / \sqrt{3}$ also induces the inequality. Hence, we prove the property.

Since PFSs are generalized form of IFSs, the distances between PFSs need to be computed by considering all the three components $\mu_{\widetilde{P}}^{2}(x), \nu_{\widetilde{P}}^{2}(x)$ and $\pi_{\widetilde{P}}^{2}(x)$ in PFSs. The well-known distance between PFSs is Euclidean distance. Therefore, the inequality in Property 10 indicates that the Euclidean distance between $\left(\mu_{\widetilde{P}}(x), \nu_{\widetilde{P}}(x), \pi_{\widetilde{P}}(x)\right)$ and $(1 / \sqrt{3}, 1 / \sqrt{3}, 1 / \sqrt{3})$ is larger than the Euclidean distance between $\left(\mu_{\widetilde{Q}}(x), \nu_{\widetilde{Q}}(x), \pi_{\widetilde{Q}}(x)\right)$ and $(1 / \sqrt{3}, 1 / \sqrt{3}, 1 / \sqrt{3})$. This manifests that $\left(\mu_{\widetilde{Q}}(x), \nu_{\widetilde{Q}}(x), \pi_{\widetilde{Q}}(x)\right)$ is located more nearby to $(1 / \sqrt{3}, 1 / \sqrt{3}, 1 / \sqrt{3})$ than that of $\left(\mu_{\widetilde{P}}(x), \nu_{\widetilde{P}}(x), \pi_{\widetilde{P}}(x)\right)$. From a geometrical perspective, the axiom $\left(A 3^{\prime}\right)$ is reasonable and logical because the closer PFS to the unique point $(1 / \sqrt{3}, 1 / \sqrt{3}, 1 / \sqrt{3})$ with maximum entropy reflects the greater entropy of that PFS.

We next construct entropies for PFSs based on a probability-type. To formulate the probability-type of entropy for PFSs, we use the idea of entropy $H^{\gamma}(p)$ of Havrda and Charavát [35] to a probability mass function $p=\left\{p_{1}, \ldots, p_{k}\right\}$ with

$$
H^{\gamma}(p)= \begin{cases}\frac{1}{\gamma-1}\left(1-\sum_{i=1}^{k} p_{i}^{\gamma}\right), & \gamma \neq 1(\gamma>0) \\ -\sum_{i=1}^{k} p_{i} \log p_{i}, & \gamma=1 .\end{cases}
$$

Let $X=\left\{x_{1}, x_{2}, \ldots, x_{n}\right\}$ be a finite universe of discourses. Thus, for a PFS $\widetilde{P}$ in $X$, we propose the following probabilitytype entropy for the PFS $\widetilde{P}$ with
Apparently, one may ask a question: "Are these proposed entropy measures for PFSs are suitable and acceptable?" To answer this question, we present the following theorem.

Theorem 11. Let $X=\left\{x_{1}, x_{2}, \ldots, x_{n}\right\}$ be a finite universe of discourses. The proposed probabilistic-type entropy $e_{\mathrm{HC}}^{\gamma}$ for a PFS $\widetilde{P}$ satisfies the axioms $(A 1),\left(A 2^{\prime}\right),\left(A 3^{\prime}\right)$, and $(A 4)$ in Definition 9.
To prove the axioms $\left(A 2^{\prime}\right)$ and $\left(A 3^{\prime}\right)$ for Theorem 11 , we need Lemma 12.

Lemma 12. Let $\psi_{\gamma}(x), 0<x<1$ be defined as

$$
\psi_{\gamma}(x)= \begin{cases}\frac{1}{\gamma-1}\left(x-x^{\gamma}\right), & \gamma \neq 1(\gamma>0) \\ -x \log x, & \gamma=1 .\end{cases}
$$

Then $\psi_{\gamma}(x)$ is a strictly concave function of $x$. 
Proof. By twice differentiating $\psi_{\gamma}(x)$, we get $\psi_{\gamma}^{\prime \prime}(x)=$ $-\gamma x^{\gamma-2}<0$, for $\gamma \neq 1(\gamma>0)$. Then $\psi_{\gamma}(x)$ is a strictly concave function of $x$. Similarly, we can show that $\psi_{\gamma=1}(x)=x \log x$ is also a strictly concave function of $x$.

Proof of Theorem 11. It is easy to check that $e_{H C}^{\gamma}$ satisfies the axioms $(A 1)$ and $(A 4)$. We need only to prove that $e_{H C}^{\gamma}$ satisfies the axioms $\left(A 2^{\prime}\right)$ and $\left(A 3^{\prime}\right)$. To prove $e_{H C}^{\gamma}$ for the case $\gamma \neq 1(\gamma>0)$ that satisfies the axiom $\left(A 2^{\prime}\right)$, we use Lagrange multipliers for $e_{H C}^{\gamma}$ with $F\left(\mu_{\widetilde{P}}^{2}, v_{\widetilde{P}}^{2}, \pi_{\tilde{P}}^{2}, \lambda\right)=(1 / n) \sum_{i=1}^{n}(1 /(\gamma-$ 1)) $\left[1-\left(\left(\mu_{\tilde{P}}^{2}\left(x_{i}\right)\right)^{\gamma}+\left(\nu_{\tilde{P}}^{2}\left(x_{i}\right)\right)^{\gamma}+\left(\pi_{\tilde{P}}^{2}\left(x_{i}\right)\right)^{\gamma}\right)\right]+\sum_{i=1}^{n} \lambda_{i}\left(\mu_{\tilde{P}}^{2}\left(x_{i}\right)+\right.$ $\left.v_{\widetilde{P}}^{2}\left(x_{i}\right)+\pi_{\widetilde{P}}^{2}\left(x_{i}\right)-1\right)$. By taking the derivative of $F\left(\mu_{\widetilde{P}}^{2}, v_{\widetilde{P}}^{2}, \pi_{\widetilde{P}}^{2}, \lambda\right)$ with respect to $\mu_{\tilde{P}}^{2}\left(x_{i}\right), v_{\tilde{P}}^{2}\left(x_{i}\right), \pi_{\tilde{P}}^{2}\left(x_{i}\right)$, and $\lambda_{i}$, we obtain

$$
\begin{aligned}
\frac{\partial F}{\partial \mu_{\widetilde{P}}^{2}\left(x_{i}\right)} & =\frac{-\gamma}{(\gamma-1)}\left(\mu_{\widetilde{P}}^{2}\left(x_{i}\right)\right)^{\gamma-1}+\lambda_{i} \stackrel{\text { set }}{=} 0, \\
\frac{\partial F}{\partial v_{\widetilde{P}}^{2}\left(x_{i}\right)} & =\frac{-\gamma}{(\gamma-1)}\left(\nu_{\widetilde{P}}^{2}\left(x_{i}\right)\right)^{\gamma-1}+\lambda_{i} \stackrel{\text { set }}{=} 0, \\
\frac{\partial F}{\partial \pi_{\widetilde{P}}^{2}\left(x_{i}\right)} & =\frac{-\gamma}{(\gamma-1)}\left(\pi_{\widetilde{P}}^{2}\left(x_{i}\right)\right)^{\gamma-1}+\lambda_{i} \stackrel{\text { set }}{=} 0, \\
\frac{\partial F}{\partial \lambda_{i}} & =\mu_{\widetilde{P}}^{2}\left(x_{i}\right)+\nu_{\widetilde{P}}^{2}\left(x_{i}\right)+\pi_{\widetilde{P}}^{2}\left(x_{i}\right)-1 \stackrel{\text { set }}{=} 0 .
\end{aligned}
$$

From above PDEs, we get $\left(\mu_{\tilde{p}}^{2}\left(x_{i}\right)\right)^{\gamma-1}=\lambda_{i}(\gamma-1) / \gamma$ and then $\mu_{\tilde{P}}^{2}\left(x_{i}\right)=\left(\lambda_{i}(\gamma-1) / \gamma\right)^{1 /(\gamma-1)} ;\left(\nu_{\tilde{P}}^{2}\left(x_{i}\right)\right)^{\gamma-1}=\lambda_{i}(\gamma-1) / \gamma$ and $\nu_{\tilde{P}}^{2}\left(x_{i}\right)=\left(\lambda_{i}(\gamma-1) / \gamma\right)^{1 /(\gamma-1)} ;\left(\pi_{\tilde{P}}^{2}\left(x_{i}\right)\right)^{\gamma-1}=\lambda_{i}(\gamma-1) / \gamma$ and $\pi_{\widetilde{P}}^{2}\left(x_{i}\right)=\left(\lambda_{i}(\gamma-1) / \gamma\right)^{1 /(\gamma-1)} ; \mu_{\widetilde{P}}^{2}\left(x_{i}\right)+\nu_{\widetilde{P}}^{2}\left(x_{i}\right)+\pi_{\widetilde{P}}^{2}\left(x_{i}\right)=1$ and then $\left(\lambda_{i}(\gamma-1) / \gamma\right)^{1 /(\gamma-1)}=1 / 3$. Thus, we have $\lambda_{i}=(\gamma /(\gamma-$ 1)) $(1 / 3)^{\gamma-1}$. We obtain $\mu_{\tilde{P}}^{2}\left(x_{i}\right)=\left((\gamma /(\gamma-1))(1 / 3)^{\gamma-1}(\gamma-\right.$ $1) / \gamma)^{1 /(\gamma-1)}$, and then $\mu_{\tilde{P}}\left(x_{i}\right)=1 / \sqrt{3}$. We also get $\nu_{\tilde{P}}\left(x_{i}\right)=$ $1 / \sqrt{3}$ and $\pi_{\tilde{P}}\left(x_{i}\right)=1 / \sqrt{3}$. That is, $\mu_{\tilde{P}}\left(x_{i}\right)=\nu_{\tilde{P}}\left(x_{i}\right)=\pi_{\tilde{P}}\left(x_{i}\right)=$ $1 / \sqrt{3}, \forall i$. Similarly, we can show that the equation of $e_{H C}^{\gamma}$ for the case $\gamma=1$ also obtains $\mu_{\tilde{P}}\left(x_{i}\right)=\nu_{\widetilde{P}}\left(x_{i}\right)=\pi_{\tilde{P}}\left(x_{i}\right)=$ $1 / \sqrt{3}, \forall i$. By Lemma 12 , we learn that the function $\psi_{\gamma}(x)$ is a strictly concave function of $x$. We know that $e_{H C}^{\gamma}(\widetilde{P})=$ $(1 / n) \sum_{i=1}^{n}\left(\psi_{\gamma}\left(\mu_{\tilde{P}}^{2}\left(x_{i}\right)\right)+\psi_{\gamma}\left(\nu_{\tilde{P}}^{2}\left(\left(x_{i}\right)\right)\right)+\psi_{\gamma}\left(\pi_{\tilde{P}}^{2}\left(x_{i}\right)\right)\right)$ and so $e_{\mathrm{HC}}^{\gamma}$ is also a strictly concave function. Therefore, it is proved that $e_{H C}^{\gamma}$ attains a unique maximum at $\mu_{\tilde{P}}\left(x_{i}\right)=v_{\widetilde{P}}\left(x_{i}\right)=$ $\pi_{\widetilde{P}}\left(x_{i}\right)=1 / \sqrt{3}, \forall i$. We next prove that the probabilistictype entropy $e_{H C}^{\gamma}$ satisfies the axiom $\left(A 3^{\prime}\right)$. If $\widetilde{P}$ is crisper than $\widetilde{Q}$, we notice that $\widetilde{P}$ is far away from $(1 / \sqrt{3}, 1 / \sqrt{3}, 1 / \sqrt{3})$ compared to $\widetilde{Q}$ according to Property 10 . However, $e_{H C}^{\gamma}$ is a strictly concave function and $e_{H C}^{\gamma}$ attains a unique maximum at $\mu_{\widetilde{P}}\left(x_{i}\right)=\nu_{\widetilde{P}}\left(x_{i}\right)=\pi_{\widetilde{P}}\left(x_{i}\right)=1 / \sqrt{3}, \forall i$. From here, we obtain $e_{H C}^{\gamma}(\widetilde{P}) \leq e_{H C}^{\gamma}(\widetilde{Q})$ if $\widetilde{P}$ is crisper than $\widetilde{Q}$. Thus, we prove the axiom $\left(A 3^{\prime}\right)$.

Concept to determine uncertainty from a fuzzy set and its complement was first given by Yager [26]. In this section, we first use the similar idea to measure uncertainty of PFSs in terms of the amount of distinction between a PFS $\widetilde{P}$ and its complement $\widetilde{P}^{c}$. However, various distance measures are made to express numerically the difference between two objects with high accuracy. Therefore, the distance between two fuzzy sets plays a vital role in theoretical and practical issues. Let $X=\left\{x_{1}, x_{2}, \ldots, x_{n}\right\}$ be a finite universe of discourses; we first define a Pythagorean normalized Euclidean (PNE) distance between two Pythagorean fuzzy sets $\widetilde{P}, \widetilde{Q} \in$ $\operatorname{PFSs}(X)$ as

$$
\begin{aligned}
\zeta_{E}(\widetilde{P}, \widetilde{Q}) & =\left[\frac { 1 } { 2 n } \sum _ { i = 1 } ^ { n } \left(\left(\mu_{\widetilde{P}}^{2}\left(x_{i}\right)-\mu_{\widetilde{Q}}^{2}\left(x_{i}\right)\right)^{2}\right.\right. \\
& \left.\left.+\left(v_{\widetilde{P}}^{2}\left(x_{i}\right)-v_{\widetilde{\mathrm{Q}}}^{2}\left(x_{k}\right)\right)^{2}+\left(\pi_{\widetilde{P}}^{2}\left(x_{i}\right)-\pi_{\widetilde{\mathrm{Q}}}^{2}\left(x_{i}\right)\right)^{2}\right)\right]^{1 / 2}
\end{aligned}
$$

We next propose fuzzy entropy induced by the PNE distance $\zeta_{E}$ between the PFS $\widetilde{P}$ and its complement $\widetilde{P}^{c}$. Let $\widetilde{P}=$ $\left\{\left\langle x_{i}, \mu_{\tilde{P}}\left(x_{i}\right), \nu_{\tilde{P}}\left(x_{i}\right)\right\rangle: x_{i} \in X\right\}$ be any Pythagorean fuzzy set on the universe of discourse $X=\left\{x_{1}, x_{2}, \ldots, x_{n}\right\}$ with its complement $\widetilde{P}^{c}=\left\{\left\langle x, v_{\widetilde{P}}\left(x_{i}\right), \mu_{\tilde{P}}\left(x_{i}\right)\right\rangle: x_{i} \in X\right\}$. The PNE distance between PFSs $\widetilde{P}$ and $\widetilde{P}^{c}$ will be $\zeta_{E}\left(\widetilde{P}, \widetilde{P}^{c}\right)=$ $\left[(1 / n) \sum_{i=1}^{n}\left(\mu_{\tilde{P}}^{2}\left(x_{i}\right)-v_{\widetilde{P}}^{2}\left(x_{i}\right)\right)^{2}\right]^{1 / 2}$. Thus, we define a new entropy $e_{E}$ for the PFS $\widetilde{P}$ as

$$
\begin{aligned}
e_{E}(\widetilde{P}) & =1-\zeta_{E}\left(\widetilde{P}, \widetilde{P}^{c}\right) \\
& =1-\sqrt{\frac{1}{n} \sum_{i=1}^{n}\left(\mu_{\widetilde{P}}^{2}\left(x_{i}\right)-v_{\widetilde{P}}^{2}\left(x_{i}\right)\right)^{2}}
\end{aligned}
$$

Theorem 13. Let $X=\left\{x_{1}, x_{2}, \ldots, x_{n}\right\}$ be a finite universe of discourse. The proposed entropy $e_{E}$ for a PFS $\widetilde{P}$ satisfies the axioms (A0) (A5) in Definition 9, and so $e_{E}$ is a $\sigma$-entropy.

Proof. We first prove the axiom (A0). Since the distance $\zeta_{E}\left(\widetilde{P}, \widetilde{P}^{c}\right)$ is between 0 and $1,0 \leq 1-\zeta_{E}\left(\widetilde{P}, \widetilde{P}^{c}\right) \leq 1$, and then $0 \leq e_{E}(\widetilde{P}) \leq 1$. The axiom (A0) is satisfied. For the axiom (A1), if $\widetilde{P}$ is crisp, i.e., $\mu_{\widetilde{P}}^{2}\left(x_{i}\right)=0, v_{\widetilde{P}}^{2}\left(x_{i}\right)=1$ or $\mu_{\tilde{P}}^{2}\left(x_{i}\right)=1, v_{\widetilde{P}}^{2}\left(x_{i}\right)=0, \forall x_{i} \in X$, then $\zeta_{E}(\widetilde{P}$, $\left.\widetilde{P}^{c}\right)=\sqrt{(1 / n) \sum_{i=1}^{n}\left(\mu_{\tilde{P}}^{2}\left(x_{i}\right)-v_{\tilde{P}}^{2}\left(x_{i}\right)\right)^{2}}=1$. Thus, we obtain $e_{E}(\widetilde{P})=1-1=0$. Conversely, if $e_{E}(\widetilde{P})=0$, then $\zeta_{E}\left(\widetilde{P}, \widetilde{P}^{c}\right)=$ $1-e_{E}(\widetilde{P})=1$, i.e., $\sqrt{(1 / n) \sum_{i=1}^{n}\left(\mu_{\widetilde{P}}^{2}\left(x_{i}\right)-v_{\widetilde{P}}^{2}\left(x_{i}\right)\right)^{2}}=1$. Thus, $\mu_{\widetilde{P}}^{2}\left(x_{i}\right)=1, v_{\widetilde{P}}^{2}\left(x_{i}\right)=0$ or $\mu_{\widetilde{P}}^{2}\left(x_{i}\right)=0, v_{\widetilde{P}}^{2}\left(x_{i}\right)=1$ and so $\widetilde{P}$ is crisp. Thus, the axiom (A1) is satisfied. Now, we prove the axiom $(\mathrm{A} 2) . \mu_{\tilde{P}}^{2}\left(x_{i}\right)=\nu_{\tilde{P}}^{2}\left(x_{i}\right), \forall x_{i} \in X$, implies that $\zeta_{E}\left(\widetilde{P}, \widetilde{P}^{c}\right)=0, e_{E}(\widetilde{P})=1$. Conversely, $e_{E}(\widetilde{P})=1$ implies $\zeta_{E}\left(\widetilde{P}, \widetilde{P}^{c}\right)=0$. Thus, we have that $\mu_{\tilde{P}}^{2}\left(x_{i}\right)=v_{\widetilde{P}}^{2}\left(x_{i}\right), \forall x_{i} \in X$. For the axiom (A3), since $\mu_{\widetilde{P}}^{2}\left(x_{i}\right) \leq \mu_{\widetilde{\mathrm{Q}}}^{2}\left(x_{i}\right)$ and $\nu_{\widetilde{P}}^{2}\left(x_{i}\right) \geq v_{\widetilde{\mathrm{Q}}}^{2}\left(x_{i}\right)$ for $\mu_{\widetilde{\mathrm{Q}}}^{2}\left(x_{i}\right) \leq \nu_{\widetilde{\mathrm{Q}}}^{2}\left(x_{i}\right)$ imply that $\mu_{\widetilde{P}}^{2}\left(x_{i}\right) \leq \mu_{\widetilde{\mathrm{Q}}}^{2}\left(x_{i}\right) \leq v_{\widetilde{\mathrm{Q}}}^{2}\left(x_{i}\right) \leq v_{\widetilde{P}}^{2}\left(x_{i}\right)$, then we have the distance $\forall x_{i} \in X, \sqrt{(1 / n) \sum_{i=1}^{n}\left(\mu_{\tilde{P}}^{2}\left(x_{i}\right)-v_{\tilde{P}}^{2}\left(x_{i}\right)\right)^{2}} \geq$ $\sqrt{(1 / n) \sum_{i=1}^{n}\left(\mu_{\widetilde{\mathrm{Q}}}^{2}\left(x_{i}\right)-\nu_{\widetilde{\mathrm{Q}}}^{2}\left(x_{i}\right)\right)^{2}}$. Again from the axiom (A3) of Definition 9, we have $\mu_{\widetilde{P}}^{2}\left(x_{i}\right) \geq \mu_{\widetilde{Q}}^{2}\left(x_{i}\right)$ and 
$v_{\widetilde{P}}^{2}\left(x_{i}\right) \leq \nu_{\widetilde{\mathrm{Q}}}^{2}\left(x_{i}\right)$ for $\mu_{\widetilde{\mathrm{Q}}}^{2}\left(x_{i}\right) \geq \nu_{\widetilde{\mathrm{Q}}}^{2}\left(x_{i}\right)$ implies that $v_{\widetilde{P}}^{2}\left(x_{i}\right) \leq \nu_{\widetilde{Q}}^{2}\left(x_{i}\right) \leq \mu_{\widetilde{Q}}^{2}\left(x_{i}\right) \leq \mu_{\widetilde{P}}^{2}\left(x_{i}\right)$, and then the distance $\forall x_{i} \in X, \sqrt{(1 / n) \sum_{i=1}^{n}\left(\mu_{\tilde{P}}^{2}\left(x_{i}\right)-v_{\tilde{P}}^{2}\left(x_{i}\right)\right)^{2}}$ $\geq \sqrt{(1 / n) \sum_{i=1}^{n}\left(\mu_{\widetilde{\mathrm{Q}}}^{2}\left(x_{i}\right)-\nu_{\widetilde{\mathrm{Q}}}^{2}\left(x_{i}\right)\right)^{2}}$. From inequalities $\sqrt{(1 / n) \sum_{i=1}^{n}\left(\mu_{\tilde{P}}^{2}\left(x_{i}\right)-v_{\widetilde{P}}^{2}\left(x_{i}\right)\right)^{2}} \geq \sqrt{(1 / n) \sum_{i=1}^{n}\left(\mu_{\widetilde{\mathrm{Q}}}^{2}\left(x_{i}\right)-v_{\widetilde{\mathrm{Q}}}^{2}\left(x_{i}\right)\right)^{2}}$ and $\quad \sqrt{(1 / n) \sum_{i=1}^{n}\left(\mu_{\tilde{P}}^{2}\left(x_{i}\right)-v_{\tilde{P}}^{2}\left(x_{i}\right)\right)^{2}}$ $\sqrt{(1 / n) \sum_{i=1}^{n}\left(\mu_{\widetilde{\mathrm{Q}}}^{2}\left(x_{i}\right)-v_{\widetilde{\mathrm{Q}}}^{2}\left(x_{i}\right)\right)^{2}}$, we have $\zeta_{E}\left(\widetilde{P}, \widetilde{P}^{c}\right) \geq \zeta_{H}(\widetilde{\mathrm{Q}}$, $\left.\widetilde{Q}^{c}\right)$, and then $1-\zeta_{E}\left(\widetilde{P}, \widetilde{P}^{c}\right) \leq 1-\zeta_{E}\left(\widetilde{Q}, \widetilde{Q}^{c}\right)$. This concludes that $e_{E}(\widetilde{P}) \leq e_{E}(\widetilde{Q})$. In this way the axiom (A3) is proved. Next, we prove the axiom (A4). Since $\forall x_{i} \in X, e_{E}(\widetilde{P})=$ $1-\zeta_{E}\left(\widetilde{P}, \widetilde{P}^{c}\right)=1-\sqrt{(1 / n) \sum_{i=1}^{n}\left(\mu_{\widetilde{P}}^{2}\left(x_{i}\right)-v_{\widetilde{P}}^{2}\left(x_{i}\right)\right)^{2}}=$ $1-\sqrt{(1 / n) \sum_{i=1}^{n}\left(v_{\tilde{P}}^{2}\left(x_{i}\right)-\mu_{\widetilde{P}}^{2}\left(x_{i}\right)\right)^{2}}, 1-\zeta_{E}\left(\widetilde{P}^{c}, \widetilde{P}\right)=e_{E}\left(\widetilde{P}^{c}\right)$. Hence, the axiom (A4) is satisfied. Finally, for proving the axiom (A5), let $\widetilde{P}$ and $\widetilde{Q}$ be two PFSs. Then, we have

(i) $\mu_{\widetilde{P}}^{2}\left(x_{i}\right) \leq \mu_{\widetilde{\mathrm{Q}}}^{2}\left(x_{i}\right)$ and $\nu_{\widetilde{P}}^{2}\left(x_{i}\right) \geq v_{\widetilde{\mathrm{Q}}}^{2}\left(x_{i}\right)$ for $\mu_{\widetilde{\mathrm{Q}}}^{2}\left(x_{i}\right) \leq$ $v_{\widetilde{\mathrm{Q}}}^{2}\left(x_{i}\right), \forall x_{i} \in X$, or

(ii) $\mu_{\widetilde{P}}^{2}\left(x_{i}\right) \geq \mu_{\widetilde{\mathrm{Q}}}^{2}\left(x_{i}\right)$ and $v_{\widetilde{P}}^{2}\left(x_{i}\right) \leq \nu_{\widetilde{\mathrm{Q}}}^{2}\left(x_{i}\right)$ for $\mu_{\widetilde{\mathrm{Q}}}^{2}\left(x_{i}\right) \geq$ $\nu_{\widetilde{Q}}^{2}\left(x_{i}\right), \forall x_{i} \in X$.

From (i), we have $\forall x_{i} \in X, \mu_{\widetilde{P}}^{2}\left(x_{i}\right) \leq \mu_{\widetilde{Q}}^{2}\left(x_{i}\right) \leq \nu_{\widetilde{Q}}^{2}\left(x_{i}\right) \leq$ $v_{\widetilde{P}}^{2}\left(x_{i}\right)$, then $\max \left(\mu_{\widetilde{P}}^{2}\left(x_{i}\right), \mu_{\widetilde{\mathrm{Q}}}^{2}\left(x_{i}\right)\right)=\mu_{\widetilde{\mathrm{Q}}}^{2}\left(x_{i}\right)$ and $\min \left(v_{\widetilde{P}}^{2}\left(x_{i}\right)\right.$, $\left.\nu_{\widetilde{\mathrm{Q}}}^{2}\left(x_{i}\right)\right)=v_{\widetilde{\mathrm{Q}}}^{2}\left(x_{i}\right)$. That is, $(\widetilde{P} \cup \widetilde{\mathrm{Q}})=\left(\mu_{\widetilde{\mathrm{Q}}}^{2}\left(x_{i}\right), v_{\widetilde{\mathrm{Q}}}^{2}\left(x_{i}\right)\right)=\widetilde{\mathrm{Q}}$ which implies that $e_{E}(\widetilde{P} \cup \widetilde{\mathrm{Q}})=e_{E}\left(\mu_{\widetilde{\mathrm{Q}}}^{2}\left(x_{i}\right), v_{\widetilde{\mathrm{Q}}}^{2}\left(x_{i}\right)\right)=e_{E}(\widetilde{\mathrm{Q}})$. Also, $\forall x_{i} \in X, \min \left(\mu_{\widetilde{P}}^{2}\left(x_{i}\right), \mu_{\widetilde{Q}}^{2}\left(x_{i}\right)\right)=\mu_{\widetilde{P}}^{2}\left(x_{i}\right)$ and $\max \left(v_{\widetilde{P}}^{2}\left(x_{i}\right)\right.$, $\left.v_{\widetilde{Q}}^{2}\left(x_{i}\right)\right)=v_{\widetilde{P}}^{2}\left(x_{i}\right)$, then $(\widetilde{P} \cap \widetilde{Q})=\left(\mu_{\widetilde{P}}^{2}\left(x_{i}\right), v_{\widetilde{P}}^{2}\left(x_{i}\right)\right)=\widetilde{P}$ implies $e_{E}(\widetilde{P} \cap \widetilde{Q})=e_{E}\left(\mu_{\widetilde{P}}^{2}\left(x_{i}\right), v_{\widetilde{P}}^{2}\left(x_{i}\right)\right)=e_{E}(\widetilde{P})$. Hence, $e_{E}(\widetilde{P})+e_{E}(\widetilde{Q})=e_{E}(\widetilde{P} \cap \widetilde{Q})+e_{E}(\widetilde{P} \cup \widetilde{Q})$. Again, from (ii), we have $\forall x_{i} \in X, v_{\widetilde{P}}^{2}\left(x_{i}\right) \leq v_{\widetilde{Q}}^{2}\left(x_{i}\right) \leq \mu_{\widetilde{Q}}^{2}\left(x_{i}\right) \leq \mu_{\widetilde{P}}^{2}\left(x_{i}\right)$. Then, $\max \left(\mu_{\widetilde{P}}^{2}\left(x_{i}\right), \mu_{\widetilde{Q}}^{2}\left(x_{i}\right)\right)=\mu_{\widetilde{P}}^{2}\left(x_{i}\right)$ and $\min \left(v_{\widetilde{P}}^{2}\left(x_{i}\right), v_{\widetilde{Q}}^{2}\left(x_{i}\right)\right)=$ $v_{\widetilde{P}}^{2}\left(x_{i}\right)$, and so $(\widetilde{P} \cup \widetilde{Q})=\left(\mu_{\widetilde{P}}^{2}\left(x_{i}\right), v_{\widetilde{P}}^{2}\left(x_{i}\right)\right)=\widetilde{P}$ which implies that $e_{E}(\widetilde{P} \cup \widetilde{Q})=e_{E}\left(\mu_{\widetilde{P}}^{2}\left(x_{i}\right), v_{\widetilde{P}}^{2}\left(x_{i}\right)\right)=e_{E}(\widetilde{P})$. Also, $\forall x_{i} \in$ $X, \min \left(\mu_{\widetilde{P}}^{2}\left(x_{i}\right), \mu_{\widetilde{Q}}^{2}\left(x_{i}\right)\right)=\mu_{\widetilde{Q}}^{2}\left(x_{i}\right)$ and $\max \left(\nu_{\widetilde{P}}^{2}\left(x_{i}\right), \nu_{\widetilde{Q}}^{2}\left(x_{i}\right)\right)=$ $v_{\widetilde{Q}}^{2}\left(x_{i}\right)$, then $(\widetilde{P} \cap \widetilde{Q})=\left(\mu_{\widetilde{Q}}^{2}\left(x_{i}\right), v_{\widetilde{Q}}^{2}\left(x_{i}\right)\right)=\widetilde{Q}$ implies that $e_{E}(\widetilde{P} \cap \widetilde{Q})=e_{E}\left(\mu_{\widetilde{Q}}^{2}\left(x_{i}\right), v_{\widetilde{Q}}^{2}\left(x_{i}\right)\right)=e_{E}(\widetilde{Q})$. Hence, $e_{E}(\widetilde{P})+$ $e_{E}(\widetilde{Q})=e_{E}(\widetilde{P} \cap \widetilde{Q})+e_{E}(\widetilde{P} \cup \widetilde{Q})$. Thus, we complete the proof of Theorem 13.

Burillo and Bustince [31] gave fuzzy entropy of intuitionistic fuzzy sets by using intuitionistic index. Now, we modify and extend the similar concept to construct the new entropy measure of PFSs by using Pythagorean index as follows.

Let $\widetilde{P}$ be a PFS on $X=\left\{x_{1}, x_{2}, \ldots, x_{n}\right\}$. We define an entropy $e_{P I}$ of $\widetilde{P}$ using Pythagorean index as

$$
\begin{aligned}
e_{P I}(\widetilde{P}) & =\frac{1}{n} \sum_{k=1}^{n}\left(1-\mu_{\widetilde{P}}^{2}\left(x_{k}\right)-\nu_{\widetilde{P}}^{2}\left(x_{k}\right)\right) \\
& =\frac{1}{n} \sum_{k=1}^{n} \pi_{\widetilde{P}}^{2}\left(x_{k}\right)
\end{aligned}
$$

Theorem 14. Let $X=\left\{x_{1}, x_{2}, \ldots, x_{n}\right\}$ be a finite universe of discourse. The proposed entropy $e_{P I}$ for the PFS $\widetilde{P}$ using Pythagorean index satisfies the axioms (A0) (A4) and (A5) in Definition 9, and so it is a $\sigma$-entropy.

Proof. Similar to Theorem 13.

We next propose a new and simple method to calculate fuzzy entropy of PFSs by using the ratio of min and max operations. All three components $\left(\mu_{\widetilde{P}}^{2}, \nu_{\widetilde{P}}^{2}, \pi_{\widetilde{P}}^{2}\right)$ of a PFS $\widetilde{P}$ are given equal importance to make the results more authentic and reliable. The new entropy is easy to be computed. Let $\widetilde{P}$ be a PFS on $X=\left\{x_{1}, x_{2}, \ldots, x_{n}\right\}$, and we define a new entropy for the PFS $\widetilde{P}$ as

$$
e_{\min / \max }(\widetilde{P})=\frac{1}{n} \sum_{i=1}^{n} \frac{\min \left(\mu_{\widetilde{P}}^{2}\left(x_{i}\right), \nu_{\widetilde{P}}^{2}\left(x_{i}\right), \pi_{\widetilde{P}}^{2}(x)\right)}{\max \left(\mu_{\widetilde{P}}^{2}\left(x_{i}\right), v_{\widetilde{P}}^{2}\left(x_{i}\right), \pi_{\widetilde{P}}^{2}(x)\right)}
$$

Theorem 15. Let $X=\left\{x_{1}, x_{2}, \ldots, x_{n}\right\}$ be a finite universe of discourse. The proposed entropy $e_{\min / \max }$ for a PFS $\widetilde{P}$ satisfies the axioms (AO) (A4) and (A5) in Definition 9, and so it is a $\sigma$-entropy.

Proof. Similar to Theorem 13.

Recently, Xue at el. [20] developed the entropy of Pythagorean fuzzy sets based on the similarity part and the hesitancy part that reflect fuzziness and uncertainty features, respectively. They defined the following Pythagorean fuzzy entropy for a PFS in a finite universe of discourses $X=$ $\left\{x_{1}, x_{2}, \ldots, x_{n}\right\}$. Let $\widetilde{P}=\left\{\left\langle x_{i}, \mu_{\widetilde{P}}\left(x_{i}\right), \nu_{\widetilde{P}}\left(x_{i}\right)\right\rangle: x_{i} \in X\right\}$ be a PFS in $X$. The Pythagorean fuzzy entropy, $E_{X u e}(\widetilde{P})$, proposed by Xue at el. [20], is defined as

$$
\begin{aligned}
& E_{\text {Xue }}(\widetilde{P}) \\
& \quad=\frac{1}{n} \sum_{i=1}^{n}\left[1-\left(\mu_{\widetilde{P}}^{2}\left(x_{i}\right)+v_{\widetilde{P}}^{2}\left(x_{i}\right)\right)\left|\mu_{\widetilde{P}}^{2}\left(x_{i}\right)-v_{\widetilde{P}}^{2}\left(x_{i}\right)\right|\right] .
\end{aligned}
$$

The entropy $E_{X u e}(\widetilde{P})$ will be compared and exhibited in next section.

\section{Examples and Comparisons}

In this section, we present simple examples to observe behaviors of our proposed fuzzy entropies for PFSs. To make it mathematically sound and practically acceptable as well as choose better entropy by comparative analysis, we give an example involving linguistic hedges. By considering linguistic example, we use various linguistic hedges like "more or less large", "quite large" "very large", "very very large", etc. in the problems under Pythagorean fuzzy environment to select 
TABLE 1: Comparison of the degree of fuzziness with different entropy measures of PFSs.

\begin{tabular}{lccccc}
\hline$P F S s$ & $e_{H C}^{1}$ & $e_{H C}^{2}$ & $e_{E}$ & $e_{P I}$ & 0.0126 \\
$\widetilde{P}$ & 0.4378 & 0.2368 & 0.2576 & 0.0146 \\
$\widetilde{Q}$ & 0.8131 & 0.5310 & 0.5700 & 0.5041 & 0.0643 \\
$\widetilde{R}$ & 1.0363 & 0.6276 & 0.7225 & 0.3527 & 0.3999 \\
\hline
\end{tabular}

TABLE 2: Degree of fuzziness from entropies of different PFSs.

\begin{tabular}{|c|c|c|c|c|c|}
\hline$P F S s$ & $e_{H C}^{1}$ & $e_{H C}^{2}$ & $e_{E}$ & $e_{P I}$ & $e_{\min / \max }$ \\
\hline$\widetilde{P}^{1 / 2}$ & 0.6407 & 0.3923 & 0.3490 & 0.2736 & 0.2013 \\
\hline$\widetilde{P}$ & 0.6066 & 0.3762 & 0.3386 & 0.3100 & 0.0957 \\
\hline$\widetilde{P}^{3 / 2}$ & 0.5375 & 0.3311 & 0.2969 & 0.3053 & 0.0542 \\
\hline$\widetilde{P}^{2}$ & 0.4734 & 0.2908 & 0.2638 & 0.2947 & 0.0233 \\
\hline$\widetilde{P}^{5 / 2}$ & 0.4231 & 0.2625 & 0.2414 & 0.2843 & 0.0108 \\
\hline$\widetilde{P}^{3}$ & 0.3848 & 0.2425 & 0.2267 & 0.2763 & 0.0050 \\
\hline
\end{tabular}

better entropy. We check the performance and behaviors of the proposed entropy measures in the environment of PFSs by exhibiting its simple intuition as follows.

Example 1. Let $\widetilde{P}, \widetilde{Q}$, and $\widetilde{R}$ be singleton element PFSs in the universe of discourse $X=\left\{x_{1}\right\}$ defined as $\widetilde{P}=$ $\left\{\left\langle x_{1}, 0.93,0.35,0.1122\right\rangle\right\}, \widetilde{Q}=\left\{\left\langle x_{1}, 0.68,0.18,0.7100\right\rangle\right\}$, and $\widetilde{R}=\left\{\left\langle x_{1}, 0.68,0.43,0.5939\right\rangle\right\}$. The numerical simulation results of entropy measures $e_{H C}^{1}, e_{H C}^{2}, e_{E}, e_{P I}$, and $e_{\min / \max }$ are shown in Table 1 for the purpose of numerical comparison. From Table 1, we can see the entropy measures of $\widetilde{R}$ almost have larger entropy than $\widetilde{P}$ and $\widetilde{Q}$ without any conflict, except $e_{P I}$. That is, the degree of uncertainty of $\widetilde{R}$ is greater than that of $\widetilde{P}$ and $\widetilde{Q}$. Furthermore, the behavior and performance of all entropies are analogous to each other, except $e_{P I}$. Apparently, all entropies $e_{H C}^{1}, e_{H C}^{2}, e_{E}$, and $e_{\min / \max }$ behave well, except $e_{P I}$.

However, in Example 1, it seems to be difficult for choosing appropriate entropy that may provide a better way to decide the fuzziness of PFSs. To overcome it, we give an example with structured linguistic variables to further analyze and compare these entropy measures in Pythagorean fuzzy environment. Thus, the following example with linguistic hedges is presented to further check behaviors and performance of the proposed entropy measures.

Example 2. Let $\widetilde{P}$ be a PFS in a universe of discourse $X=$ $\{1,3,5,7,9\}$ defined as $\widetilde{P}=\{\langle 1,0.1,0.8\rangle,\langle 3,0.4,0.7\rangle$, $\langle 5,0.5,0.3\rangle,\langle 7,0.9,0.0\rangle,\langle 9,1.0,00\rangle\}$. By Definitions 6,7 , and 8 where the concentration and dilation of $\widetilde{P}$ are defined as Concentration: $\operatorname{CON}(\widetilde{P})=\widetilde{P}^{2}$, Dilation: $\operatorname{DIL}(\widetilde{P})^{1 / 2}$. By considering the characterization of linguistic variables, we use the PFS $\widetilde{P}$ to define the strength of the structural linguistic variable $\widetilde{P}$ in $X=\{1,3,5,7,9\}$. Using above defined operators, we consider the following:

$\widetilde{P}^{1 / 2}$ is regarded as "More or less LARGE"; $\widetilde{P}$ is regarded as "LARGE";

$\widetilde{P}^{3 / 2}$ is regarded as "Quite LARGE"; $\widetilde{P}^{2}$ is regarded as "Very LARGE";
$\widetilde{P}^{5 / 2}$ is regarded as "Quite very LARGE"; $\widetilde{P}^{3}$ is regarded as "Very very LARGE".

We use above linguistic hedges for PFSs to compare the entropy measures $e_{H C}^{1}, e_{H C}^{2}, e_{E}, e_{P I}$, and $e_{\min / \max }$, respectively. From intuitive point of view, the following requirement of (18) for a good entropy measure should be followed:

$$
\begin{aligned}
e\left(\widetilde{P}^{1 / 2}\right) & >e(\widetilde{P})>e\left(\widetilde{P}^{3 / 2}\right)>e\left(\widetilde{P}^{2}\right)>e\left(\widetilde{P}^{5 / 2}\right) \\
& >e\left(\widetilde{P}^{3}\right) .
\end{aligned}
$$

After calculating these entropy measures $e_{H C}^{1}, e_{H C}^{2}, e_{E}, e_{P I}$, and $e_{\min / \max }$ for these PFSs, the results are shown in Table 2. From Table 2, it can be seen that these entropy measures $e_{H C}^{1}, e_{H C}^{2}, e_{E}$, and $e_{\min / \max }$ satisfy the requirement of (18), but $e_{P I}$ fails to satisfy (18) that has $e_{P I}(\widetilde{P})>e_{P I}\left(\widetilde{P}^{3 / 2}\right)>e_{P I}\left(\widetilde{P}^{2}\right)>$ $e_{P I}\left(\widetilde{P}^{5 / 2}\right)>e_{P I}\left(\widetilde{P}^{3}\right)>e_{P I}\left(\widetilde{P}^{1 / 2}\right)$. Therefore, we say that the behaviors of $e_{H C}^{1}, e_{H C}^{2}, e_{E}$, and $e_{\min / \max }$ are good, but $e_{P I}$ is not.

In order to make more comparisons of entropy measures, we shake the degree of uncertainty of the middle value " 5 " in $X$. We decrease the degree of uncertainty for the middle point in $X$, and then we observe the amount of changes and also the impact of entropy measures when the degree of uncertainty of the middle value in $\mathrm{X}$ is decreasing. To observe how different PFS “LARGE" in X affects entropy measures, we modify $\widetilde{P}$ as

$$
\begin{gathered}
\text { “LARGE" }=\widetilde{P}_{1}=\{\langle 1,0.1,0.8\rangle,\langle 3,0.4,0.7\rangle, \\
\langle 5,0.6,0.5\rangle,\langle 7,0.9,0.0\rangle,\langle 9,1.0,00\rangle\}
\end{gathered}
$$

Again, we use PFSs $\widetilde{P}_{1}^{1 / 2}, \widetilde{P}_{1}, \widetilde{P}_{1}^{3 / 2}, \widetilde{P}_{1}^{2}, \widetilde{P}_{1}^{5 / 2}$, and $\widetilde{P}_{1}^{3}$ with linguistic hedges to compare and observe behaviors of entropy measures. The results of degree of fuzziness for different PFSs from entropy measures are shown in Table 3. From Table 3, we can see that these entropies $e_{H C}^{1}, e_{E}$, and $e_{\min / \max }$ satisfy the requirement of (18), but $e_{H C}^{2}$ and $e_{P I}$ could not fulfill the requirement of $(18)$ with $e_{H C}^{2}(\widetilde{P})>e_{H C}^{2}\left(\widetilde{P}^{1 / 2}\right)>e_{H C}^{2}\left(\widetilde{P}^{3 / 2}\right)>$ $e_{H C}^{2}\left(\widetilde{P}^{2}\right)>e_{H C}^{2}\left(\widetilde{P}^{5 / 2}\right)>e_{H C}^{2}\left(\widetilde{P}^{3}\right)$ and $e_{P I}(\widetilde{P})>\left(\widetilde{P}^{3 / 2}\right)>$ $e_{P I}\left(\widetilde{P}^{1 / 2}\right)>e_{P I}\left(\widetilde{P}^{2}\right)>e_{P I}\left(\widetilde{P}^{5 / 2}\right)>e_{P I}\left(\widetilde{P}^{3}\right)$. Therefore, the 
Table 3: Degree of fuzziness from entropies of different PFSs.

\begin{tabular}{lccccc}
\hline$P F S s$ & $e_{H C}^{1}$ & $e_{H C}^{2}$ & $e_{E}$ & $e_{P I}$ & 0.2360 \\
$\widetilde{P}_{1}^{1 / 2}$ & 0.6569 & 0.3952 & 0.3473 & 0.2560 & 0.2276 \\
$\widetilde{P}_{1}^{1}$ & 0.6554 & 0.4086 & 0.3406 & 0.2440 \\
$\widetilde{P}_{1}^{3 / 2}$ & 0.5997 & 0.3757 & 0.2944 & 0.2281 & 0.1966 \\
$\widetilde{P}_{1}^{2}$ & 0.5351 & 0.3356 & 0.2526 & 0.2145 & 0.0662 \\
$\widetilde{P}_{1}^{5 / 2}$ & 0.4753 & 0.2993 & 0.2209 & 0.2038 \\
$\widetilde{P}_{1}^{3}$ & 0.4233 & 0.2682 & 0.1976 & 0.0171 \\
\hline
\end{tabular}

TABLE 4: Degree of fuzziness from entropies of different PFSs.

\begin{tabular}{lcccc}
\hline$P F S s$ & $E_{X u e}$ & $e_{H C}^{1}$ & $e_{E}$ & $e_{\min / \max }$ \\
\hline$\widetilde{P}$ & 0.4718 & 0.7532 & 0.3603 & 0.1270 \\
$\widetilde{Q}$ & 0.4718 & 0.6886 & 0.3066 & 0.0470 \\
$\widetilde{R}$ & 0.4718 & 0.7264 & 0.4241 & 0.1111 \\
\hline
\end{tabular}

performance of $e_{H C}^{1}, e_{E}$, and $e_{\min / \max }$ is good, and $e_{H C}^{2}$ is not good, but $e_{P I}$ presents very poor. We see that a little change in uncertainty for the middle value in $\mathrm{X}$ did not affect entropies $e_{H C}^{1}, e_{E}$, and $e_{\min / \max }$, and it brings a slight change in $e_{H C}^{2}$, but it gives an absolute big effect for entropy $e_{P I}$.

In viewing the results from Tables 1,2 , and 3 , we may say that entropy measures $e_{H C}^{1}, e_{E}$, and $e_{\min / \max }$ present better performance. On the other hand, from the viewpoint of structured linguistic variables, we see that entropy measures $e_{H C}^{1}, e_{E}$, and $e_{\min / \max }$ are more suitable, reliable, and well suited in Pythagorean fuzzy environment for exhibiting the degree of fuzziness of PFS. We, therefore, recommend these entropies $e_{H C}^{1}, e_{E}$, and $e_{\min / \max }$ in a subsequent application involving multicriteria decision making.

In the following example, we conduct the comparison analysis of proposed entropies $e_{H C}^{1}, e_{E}$, and $e_{\min / \max }$ with the entropy $E_{X u e}(\widetilde{P})$, developed by Xue at el. [20], to demonstrate the advantages of our developed entropies $e_{H C}^{1}, e_{E}$, and $e_{\min / \max }$.

Example 3. Let $\widetilde{P}, \widetilde{Q}$, and $\widetilde{R}$ be PFSs in the singleton universe set $X=\left\{x_{1}\right\}$ as

$$
\begin{aligned}
& \widetilde{P}=\left\{\left\langle x_{1}, 0.305,856,0.4174\right\rangle\right\}, \\
& \widetilde{Q}=\left\{\left\langle x_{1}, 0.1850,0.8530,0.4880\right\rangle\right\}, \\
& \widetilde{R}=\left\{\left\langle x_{1}, 0.4130,0.8640,0.2880\right\rangle\right\} .
\end{aligned}
$$

The degrees of entropy for different PFSs between the proposed entropies $e_{H C}^{1}, e_{E}, e_{\min / \max }$ and the entropy $E_{X u e}(\widetilde{P})$ by Xue at el. [20] are shown in Table 4. As can be seen from Table 4, we find that despite having three different PFSs, the entropy measure $E_{X u e}$ could not distinguish the PFSs $\widetilde{P}, \widetilde{Q}$, and $\widetilde{R}$. However, the proposed entropy measures $e_{H C}^{1}, e_{E}$, and $e_{\min / \max }$ can actually differentiate these different PFSs $\widetilde{P}, \widetilde{Q}$, and $\widetilde{R}$.

\section{Pythagorean Fuzzy Multicriterion Decision Making Based on New Entropies}

In this section, we construct a new multicriterion decision making method. Specifically, we extend the technique for order preference by similarity to an ideal solution (TOPSIS) to multicriterion decision making, based on the proposed entropy measures for PFSs. Impreciseness and vagueness is a reality of daily life which requires close attentions in the matters of management and decision. In real life setting with decision making process, information available is often uncertain, vague, or imprecise. PFSs are found to be a powerful tool to solve decision making problems involving uncertain, vague, or imprecise information with high precision. To display practical reasonability and validity, we apply our proposed new entropies $e_{H C}^{1}, e_{E}$, and $e_{\min / \max }$ in a multicriteria decision making problem, involving unknown information about criteria weights for alternatives in Pythagorean fuzzy environment.

We formalize the problem in the form of decision matrix in which it lists various project alternatives. We assume that there are $m$ project alternatives and we want to compare them on $n$ various criteria $C_{j}, j=1,2, \ldots, n$. Suppose, for each criterion, we have an evaluation value. For instance, the first project on the first criterion has an evaluation $x_{11}$. The first project on the second criterion has an evaluation $x_{12}$, and the first project on $n$th criterion has an evaluation $x_{1 n}$. Our objective is to have these evaluations on individual criteria and come up with a consolidated value for the project 1 and do something similar to the project 2 and so on. We then ultimately obtain a value for each of the projects. Finally, we can rank the projects with selecting the best one among all projects.

Let $A=\left\{A_{1}, A_{2}, \ldots, A_{m}\right\}$ be the set of alternatives, and let the set of criteria for the alternatives $A_{i}, i=1,2, \ldots, m$ be represented by $C_{j}, j=1,2, \ldots, n$. The aim is to choose the best alternative out of the $n$ alternatives. The construction steps for the new Pythagorean fuzzy TOPSIS based on the proposed entropy measures are as follows. 
Step 1 (construction of Pythagorean fuzzy decision matrix). Consider that the alternative $A_{i}$ acting on the criteria $C_{j}$ is represented in terms of Pythagorean fuzzy value $\widetilde{b}_{i j}=\left(\mu_{i j}, v_{i j}\right.$, $\left.\pi_{i j}\right)_{p}, i=1,2, \ldots, m, j=1,2, \ldots, n$, where $\mu_{i j}$ denotes the degree of fulfillment, $v_{i j}$ represents the degree of not fulfillment, and $\pi_{i j}$ represents the degree of hesitancy against the

$$
\widetilde{D}=\left(\widetilde{b}_{i j}\right)_{m \times n}=\begin{gathered}
C_{1} \\
A_{1} \\
A_{2} \\
\vdots \\
A_{m}
\end{gathered}\left[\begin{array}{c}
\left(\mu_{11}, \nu_{11}, \pi_{11}\right)_{p} \\
\left(\mu_{21}, v_{21}, \pi_{21}\right)_{p} \\
\vdots \\
\left(\mu_{m 1}, v_{m 1}, \pi_{m 1}\right)_{p}
\end{array}\right.
$$

Step 2 (determination of the weights of criteria). In this step, the crux to the problem is that weights to criteria have to be identified. The weights or priorities can be obtained by different ways. Suppose that the criteria information weights are unknown and therefore, the weights $w_{j}, j=1,2,3, \ldots, n$ of criteria for Pythagorean fuzzy entropy measures can be obtained by using $e_{H C}^{1}, e_{E}$, and $e_{\min / \max }$, respectively. Suppose the weights of criteria $C_{j}, j=1,2, \ldots, n$ are $w_{j}, j=$ $1,2,3, \ldots, n$ with $0 \leq w_{j} \leq 1$ and $\sum_{j=1}^{n} w_{j}=1$. Since weights of criteria are completely unknown, we propose a new entropy weighting method based on the proposed Pythagorean fuzzy entropy measures as follows:

$$
w_{j}=\frac{\widetilde{E}_{j}}{\sum_{j=1}^{n} \widetilde{E}_{j}}
$$

where the weights of the criteria $C_{j}$ is calculated with $\widetilde{E}_{j}=$ $(1 / m) \sum_{i=1}^{m} \widetilde{b}_{i j}$.

Step 3 (Pythagorean fuzzy positive-ideal solution (PFPIS) and Pythagorean fuzzy negative-ideal solution (PFNIS)). In general, it is important to determine the positive-ideal solution (PIS) and negative-ideal solution (NIS) in a TOPSIS method. Since the evaluation criteria can be categorized into two categories, benefit and cost criteria in TOPSIS, let $M_{1}$ and $M_{2}$ be the sets of benefit criteria and cost criteria in criteria $C_{j}$, respectively. According to Pythagorean fuzzy sets and the principle of a TOPSIS method, we define a Pythagorean fuzzy PIS (PFPIS) as follows:

$$
A^{+}=\left\{\left\langle C_{j},\left(\mu_{j}^{+}, \nu_{j}^{+}, \pi_{j}^{+}\right)\right\rangle\right\},
$$

where $\left(\mu_{j}^{+}, v_{j}^{+}, \pi_{j}^{+}\right)=(1,0,0)$,

$$
\left(\mu_{j}^{-}, \nu_{j}^{-}, \pi_{j}^{-}\right)=(0,1,0) \text {, }
$$

$$
j \in M_{1}
$$

Similarly, a Pythagorean fuzzy NIS (PFNIS) is defined as

$$
A^{-}=\left\{\left\langle C_{j},\left(\mu_{j}^{-}, \nu_{j}^{-}, \pi_{j}^{-}\right)\right\rangle\right\},
$$

alternative $A_{i}$ to the criteria $C_{j}$ with the following conditions: $0 \leq \mu_{i j}^{2} \leq 1,0 \leq v_{i j}^{2} \leq 1,0 \leq \pi_{i j}^{2} \leq 1$, and $\mu_{i j}^{2}+v_{i j}^{2}+\pi_{i j}^{2}=1$. The decision matrix $\widetilde{D}=\left(\widetilde{b}_{i j}\right)_{m \times n}$ is constructed to handle the problems involving multicriterion decision making, where the decision matrix $\widetilde{D}=\left(\widetilde{b}_{i j}\right)_{m \times n}$ can be constructed as follows:

$\left.\begin{array}{ccc}C_{2} & \cdots & C_{n} \\ \left(\mu_{12}, v_{12}, \pi_{12}\right)_{p} & \ldots & \left(\mu_{1 n}, v_{1 n}, \pi_{1 n}\right)_{p} \\ \left(\mu_{22}, v_{22}, \pi_{22}\right)_{p} & \ldots & \left(\mu_{2 n}, v_{2 n}, \pi_{2 n}\right)_{p} \\ \vdots & \vdots & \vdots \\ \left(\mu_{m 2}, v_{m 2}, \pi_{m 2}\right)_{p} & \cdots & \left(\mu_{m n}, v_{m n}, \pi_{m n}\right)_{p}\end{array}\right]$

$$
\begin{array}{r}
\text { where }\left(\mu_{j}^{-}, \nu_{j}^{-}, \pi_{j}^{-}\right)=(0,1,0), \\
\left(\mu_{j}^{+}, v_{j}^{+}, \pi_{j}^{+}\right)=(1,0,0),
\end{array}
$$$$
j \in M_{2}
$$

Step 4 (calculation of distance measures from PFPIS and PFNIS). In this step, we need to use a distance between two PFSs. Following the similar idea from the previously defined PNE distance $\zeta_{E}$ between two PFSs, we define a Pythagorean weighted Euclidean (PWE) distance for any two PFSs $\widetilde{P}, \widetilde{Q} \epsilon$ $\operatorname{PFS}(X)$ as

$$
\begin{aligned}
& \zeta_{w E}(\widetilde{P}, \widetilde{Q})=\left[\frac { 1 } { 2 } \sum _ { i = 1 } ^ { n } w _ { i } \left(\left(\mu_{\widetilde{P}}^{2}\left(x_{i}\right)-\mu_{\widetilde{Q}}^{2}\left(x_{i}\right)\right)^{2}\right.\right. \\
& \left.\left.+\left(\nu_{\widetilde{P}}^{2}\left(x_{i}\right)-v_{\widetilde{\mathrm{Q}}}^{2}\left(x_{i}\right)\right)^{2}+\left(\pi_{\widetilde{P}}^{2}\left(x_{i}\right)-\pi_{\widetilde{\mathrm{Q}}}^{2}\left(x_{i}\right)\right)^{2}\right)\right]^{1 / 2}
\end{aligned}
$$

We next use the PWE distance $\zeta_{w E}$ to calculate the distances $\widetilde{D}^{+}\left(A_{i}\right)$ and $\widetilde{D}^{-}\left(A_{i}\right)$ of each alternative $A_{i}$ from PFPIS and PFNIS, respectively, as follows:

$$
\begin{aligned}
& \widetilde{D}^{+}\left(A_{i}\right)=\widetilde{d}_{E}\left(\mathrm{~A}_{i}, \mathrm{~A}^{+}\right) \\
& =\sqrt{\frac{1}{2} \sum_{j=1}^{n} w_{j}\left[\left(1-\mu_{i j}^{2}\right)^{2}+\left(v_{i j}^{2}\right)^{2}+\left(1-\mu_{i j}^{2}-v_{i j}^{2}\right)^{2}\right]} \\
& \widetilde{D}^{-}\left(A_{i}\right)=\widetilde{d}_{E}\left(\mathrm{~A}_{i}, \mathrm{~A}^{-}\right) \\
& =\sqrt{\frac{1}{2} \sum_{j=1}^{n} w_{j}\left[\left(\mu_{i j}^{2}\right)^{2}+\left(1-v_{i j}^{2}\right)^{2}+\left(1-\mu_{i j}^{2}-v_{i j}^{2}\right)^{2}\right]}
\end{aligned}
$$

Step 5 (calculation of relative closeness degree and ranking of alternatives). The relative closeness degree $\widetilde{N}\left(A_{i}\right)$ of each 
TABle 5: Criteria to evaluate an audit company.

\begin{tabular}{ll}
\hline Criteria & Description of criteria \\
\hline $\begin{array}{l}\text { Required experience and capability to } \\
\text { make independent decision }\left(C_{1}\right)\end{array}$ & $\begin{array}{l}\text { Certification and required knowledge on accounting business and taxation law, } \\
\text { understanding of management system, auditor should not be trembled or influence }\end{array}$ \\
by capyobility to comprehend different & $\begin{array}{l}\text { Ability to work with different companies setups, analytical ability, planning and } \\
\text { strategy. } \\
\text { business needs }\left(C_{2}\right)\end{array}$ \\
Eastered excellent communication skills, well versed in compelling report, \\
convincing skills to present their reports, should be patient enough to elaborate \\
points to the entire satisfaction of the auditee.
\end{tabular}

TABle 6: Pythagorean fuzzy decision matrices.

\begin{tabular}{lccr}
\hline Alternatives & Criteria Evalution* & $C_{2}$ & $C_{3}$ \\
\hline$A_{1}$ & $C_{1}$ & $(0.6030,0.51400,0.6101)$ & $(0.5000,0.1000,0.8602)$ \\
$A_{2}$ & $(0.5500,0.4130,0.7259)$ & $(0.4000,0.2000,0.8944)$ & $(0.4500,0.5050,0.7365)$ \\
$A_{3}$ & $(0.4000,0.2000,0.8944)$ & $(0.6030,0.51400,0.6101)$ & $(0.5500,0.4130,0.7259)$ \\
\hline
\end{tabular}

TABLE 7: Weight of criteria.

\begin{tabular}{lccc}
\hline & $w_{1}$ & $w_{2}$ & $w_{3}$ \\
\hline$E_{\text {Xue }}$ & 0.3333 & 0.3333 & 0.3333 \\
$e_{H C}^{1}$ & 0.2912 & 0.3646 & 0.3442 \\
$e_{\min / \max }$ & 0.2209 & 0.4640 & 0.3151 \\
\hline
\end{tabular}

alternative $A_{i}$ with respect to PFPIS and PFNIS is obtained by using the following expression:

$$
\widetilde{N}\left(A_{i}\right)=\frac{\widetilde{D}^{-}\left(A_{i}\right)}{\widetilde{D}^{-}\left(A_{i}\right)+\widetilde{D}^{+}\left(A_{i}\right)}
$$

Finally, the alternatives are ordered according to the relative closeness degrees. The larger value of the relative closeness degrees reflects that an alternative is closer to PFPIS and farther from PFNIS, simultaneously. Therefore, the ranking order of all alternatives can be determined according to ascending order of the relative closeness degrees. The most preferred alternative is the one with the highest relative closeness degree.

In the next example, we present a comparison between the proposed entropies $e_{H C}^{1}$ and $e_{\min / \max }$ with the entropy $E_{X u e}$ by Xue at el. [20] based on PFSs for multicriteria decision making problem. The prime objective of decision makers is to select a best alternative from a set of available alternatives according to some criteria in multicriteria decision making process. Corruption is the misuse and mishandle of public power and resources for private and individual interest and benefits, usually in the form of bribery and favouritism. In addition, corruption twists and manipulates the basis of competitions by misallocating resources and slowing economic activity (Wikipedia).

Example 4. In this example, a real world problem on selection of well renowned national or international audit company is taken into account to demonstrate the comparison analysis among the proposed probabilistic entropy $e_{H C}^{1}$ and nonprobabilistic entropy $e_{\min / \max }$ with the entropy $E_{X u e}$ [20]. To ensure the transparency and accountability of state run intuitions, the ministry of finance of a developing country offers quotations to select a renowned audit company to get unbiased and fair audit report to keep on tract the economic development of a state. The quotations which are gone through scrutiny process and found successful by a committee are comprised of experts. The quotations which are found successfully by the committee are called eligible while the rest are rejected. A commission of experts is invited to rank the audit companies $\left\{A_{1}, A_{2}, A_{3}\right\}$ and to select the best one on the basis of set criteria $\left\{C_{1}, C_{2}, C_{3}\right\}$. The descriptions about criteria are given in Table 5, and Pythagorean fuzzy decision matrices are presented in Table 6. The obtained weights of criteria from the entropies $e_{H C}^{1}$, $e_{\min / \max }$, and $E_{X u e}$ are shown in Table 7. From Table 7, it is seen that the weights of criteria obtained by the entropy $E_{X u e}$ are always the same despite having different alternatives. However, the proposed entropies $e_{H C}^{1}$ and $e_{\min / \max }$ correctly differentiate the weights of criteria for each alternative $A_{i}$. The weights of criteria in Table 7 are also used to calculate the distances $\widetilde{D}^{+}\left(A_{i}\right)$ and $\widetilde{D}^{-}\left(A_{i}\right)$ of each alternative $A_{i}$ from PFPIS and PFNIS, respectively, where the results are shown in Table 8. Furthermore, the relative closeness degrees of each alternative to ideal solution are shown in Table 9. It can be seen that the relative closeness degrees obtained by the proposed entropies $e_{H C}^{1}$ and $e_{\min / \max }$ are different for different alternatives $A_{i}$, but the relative closeness degrees obtained by the entropy $E_{X u e}$ [20] could not differentiate different 
TABLE 8: Distance for each alternative.

\begin{tabular}{lcccccccc}
\hline$E_{\text {Xue }}$ & $D^{-}\left(A_{i}\right)$ & $D^{+}\left(A_{i}\right)$ & $e_{H C}^{1}$ & $D^{-}\left(A_{i}\right)$ & $D^{+}\left(A_{i}\right)$ & $e_{\min / \max }$ & $D^{-}\left(A_{i}\right)$ & $D^{+}\left(A_{i}\right)$ \\
\hline$A_{1}$ & 0.7593 & 0.6476 & $A_{1}$ & 0.7587 & 0.6468 & $A_{1}$ & 0.7455 & 0.6363 \\
$A_{2}$ & 0.8230 & 0.7842 & $A_{2}$ & 0.8208 & 0.7830 & $A_{2}$ & 0.8269 & 0.7862 \\
$A_{3}$ & 0.7593 & 0.6476 & $A_{3}$ & 0.7493 & 0.6403 & $A_{3}$ & 0.7284 & 0.6244 \\
\hline
\end{tabular}

TABLE 9: Degree of relative closeness.

\begin{tabular}{lllccr}
\hline$E_{\text {Xue }}$ & $N\left(A_{i}\right)$ & $e_{H C}^{1}$ & $N\left(A_{i}\right)$ & $e_{\min / \max }$ & $N\left(A_{i}\right)$ \\
\hline$A_{1}$ & 0.5397 & $A_{1}$ & 0.5398 & $A_{1}$ & 0.5395 \\
$A_{2}$ & 0.5121 & $A_{2}$ & 0.5118 & $A_{2}$ & 0.5126 \\
$A_{3}$ & 0.5397 & $A_{3}$ & 0.5392 & $A_{3}$ & 0.5384 \\
\hline
\end{tabular}

TABLE 10: Ranking of alternatives by different methods.

\begin{tabular}{lcc}
\hline Method & Ranking & Best alternative \\
\hline$E_{X u e}$ & $A_{2} \prec A_{1}=A_{3}$ & None \\
$e_{H C}^{1}$ & $A_{2} \prec A_{3} \prec A_{1}$ & $A_{1}$ \\
$e_{\min / \max }$ & $A_{2} \prec A_{3} \prec A_{1}$ & $A_{1}$ \\
\hline
\end{tabular}

alternatives so that it gives bias ranking of alternatives. These ranking results of different alternatives by the entropies $e_{H C}^{1}$, $e_{\min / \max }$ and $E_{X u e}$ are shown in Table 10. As can be seen, the ranking of alternatives by the proposed entropies $e_{H C}^{1}$ and $e_{\min / \max }$ is well; however, the entropy $E_{X u e}$ [20] could not rank the alternative $A_{1}$ and $A_{3}$. It is found that there is no conflict in ranking alternatives by using the proposed Pythagorean fuzzy TOPSIS method based on the proposed entropies $e_{H C}^{1}$ and $e_{\min / \max }$. Totally, the comparative analysis shows that the best alternative is $A_{1}$.

We next apply the constructed Pythagorean fuzzy TOPSIS in multicriterion decision making for China-Pakistan Economic Corridor projects.

Example 5. A case study in ranking China-Pakistan Economic Corridor projects on priorities basis in the light of related experts' opinions is used in order to demonstrate the efficiency of the proposed Pythagorean fuzzy TOPSIS being applied to multicriterion decision making. China-Pakistan Economic Corridor (CPEC) is a collection of infrastructure projects that are currently under construction throughout in Pakistan. Originally valued at $\$ 46$ billion, the value of CPEC projects is now worth $\$ 62$ billion. CPEC is intended to rapidly modernize Pakistani infrastructure and strengthen its economy by the construction of modern transportation networks, numerous energy projects, and special economic zones. CPEC became partly operational when Chinese cargo was transported overland to Gwadar Port for onward maritime shipment to Africa and West Asia (see Wikipedia). It is not only to benefit China and Pakistan, but also to have positive impact on other countries and regions. Under CPEC projects, it will have more frequent and free exchanges of growth, people to people contacts, and integrated region of shared destiny by enhancing understanding through academic, cultural, regional knowledge, and activity of higher volume of flows in trades and businesses. The enhancement of geographical linkages and cooperation by a win-win model will result in improving the life standard of people, road, rail, and air transportation systems and also sustainable and perpetual development in China and Pakistan.

Now suppose the concern and relevant experts are allowed to rank the CPEC projects according to needs of both countries on priorities basis. Assume that initially there are five mega projects which are Gwadar Port $\left(A_{1}\right)$, Infrastructure $\left(\mathrm{A}_{2}\right)$, Economic Zones $\left(\mathrm{A}_{3}\right)$, Transportation and Energy $\left(\mathrm{A}_{4}\right)$, and Social Sector Development $\left(\mathrm{A}_{5}\right)$, according to the following four criteria: time frame and infrastructural improvement $\left(C_{1}\right)$, maintenance and sustainability $\left(C_{2}\right)$, socioeconomic development $\left(\mathrm{C}_{3}\right)$, and eco-friendly $\left(\mathrm{C}_{4}\right)$. A detailed description of such criteria is displayed in Table 11. Consider a decision organization with the five concerns, where relevant experts are authorized to rank the satisfactory degree of an alternative with respect to the given criterion, which is represented by a Pythagorean fuzzy value (PFV). The evaluation values of the five alternatives $A_{i}, i=1,2, \ldots, 5$ with Pythagorean fuzzy decision matrix are given in Table 12.

We next use entropy measures $e_{H C}^{1}, e_{E}$, and $e_{\min / \max }$ based on better performance in numerical analysis to calculate the criteria weights $w_{j}$ using (22). These results are shown in Table 13. From Table 13, we can see that the criteria weights and ranking of weights obtained by each entropy measure are different. We find the distances $\widetilde{D}^{+}$and $\widetilde{D}^{-}$of each alternative from PFPIS and PFNIS using (23) and (24). The results are shown in Table 14. We also calculate the relative closeness degrees $\widetilde{N}$ of alternatives using (27). The results are shown in Table 15. From Table 15, it can be clearly seen that, under different entropy measures, the relative closeness degrees of alternatives obtained are different, but the gap between these values are considerably small. Thus, the ranking of alternatives is almost the same. The final ranking results from different entropies are shown in Table 16. From Table 16, it can be identified that there is no conflict in selecting the best alternative among alternatives by using the proposed Pythagorean fuzzy TOPSIS method based on the entropies $e_{H C}^{1}, e_{E}$, and $e_{\min / \max }$. There is only one conflict to be found in deciding the preference ordering of alternatives $A_{4}$ and $A_{5}$ in $e_{E}$. Hence, the results of ranking of alternatives according to 
TABLE 11: Criteria to assess CPEC projects.

\begin{tabular}{ll}
\hline Criterion & Description of criterion \\
\hline $\begin{array}{l}\text { Time frame and infrastructural } \\
\text { improvement } C_{1}\end{array}$ & $\begin{array}{l}\text { Roadmap to ensure timely completion of project without any interruption and } \\
\text { hurdle and play a vital role in making infrastructural improvement and } \\
\text { development }\end{array}$ \\
Maintenance and sustainability $C_{2}$ & $\begin{array}{l}\text { The maintenance, repair, reliability and sustainability of the project } \\
\text { Bring visible development and improvement in GDP, economy stability and } \\
\text { prosperity, life expectancy, education, health, employment, personal dignity, } \\
\text { personal safety and freedom } \\
\text { Socioeconomic development } C_{3}\end{array}$ \\
Eco - friendly $C_{4}$ & $\begin{array}{l}\text { Not harmful to the environment, contributes to green living, practices that help } \\
\text { conserve natural resources and prevent contribution to air, water and land pollution. }\end{array}$ \\
\hline
\end{tabular}

TABLE 12: Pythagorean fuzzy decision matrix.

\begin{tabular}{lcccc}
\hline Alternatives & Criteria Evaluation & & & \\
& $C_{1}$ & $C_{2}$ & $(0.35,0.70,0.6225)$ & $C_{4}$ \\
\hline$A_{1}$ & $(0.60,0.50,0.6245)$ & $(0.65,0.45,0.6124)$ & $(0.50,0.70,0.5099)$ \\
$A_{2}$ & $(0.80,0.40,0.4472)$ & $(0.80,0.40,0.4472)$ & $(0.70,0.30,0.6481)$ & $(0.60,0.30,0.7416)$ \\
$A_{3}$ & $(0.60,0.50,0.6245)$ & $(0.70,0.50,0.5099)$ & $(0.70,0.35,0.6225)$ & $(0.40,0.20,0.8944)$ \\
$A_{4}$ & $(0.90,0.30,0.3162)$ & $(0.80,0.35,0.4873)$ & $(0.50,0.30,0.8124)$ & $(0.20,0.50,0.8426)$ \\
$A_{5}$ & $(0.80,0.40,0.4472)$ & $(0.50,0.30,0.8124)$ & $(0.70,0.50,0.5099)$ & $(0.60,0.50,0.6245)$ \\
\hline
\end{tabular}

TABLE 13: Entropies and weights of the criteria.

\begin{tabular}{lcccc}
\hline & $w_{1}$ & $w_{2}$ & $w_{3}$ & $w_{4}$ \\
\hline$e_{H C}^{1}$ & 0.2487 & 0.2563 & 0.2585 & 0.2366 \\
$e_{E}$ & 0.2063 & 0.2411 & 0.2539 & 0.2987 \\
$e_{\min / \max }$ & 0.3048 & 0.2524 & 0.2141 & 0.2288 \\
\hline
\end{tabular}

TABLE 14: Distance for each alternative.

\begin{tabular}{cccccccc}
\hline$e_{H C}^{1}$ & $D^{-}\left(A_{i}\right)$ & $D^{+}\left(A_{i}\right)$ & $e_{E}$ & $D^{-}\left(A_{i}\right)$ & $D^{+}\left(A_{i}\right)$ & $e_{\min / \max }$ & $D^{-}\left(A_{i}\right)$ \\
\hline$A_{1}$ & 0.5732 & 0.6297 & $A_{1}$ & 0.5608 & 0.6354 & $A_{1}$ & 0.5825 \\
$A_{2}$ & 0.7757 & 0.4381 & $A_{2}$ & 0.7776 & 0.4557 & $A_{2}$ & 0.7741 \\
$A_{3}$ & 0.7445 & 0.5848 & $A_{3}$ & 0.7592 & 0.6055 & $A_{3}$ & 0.7377 \\
$A_{4}$ & 0.8012 & 0.5819 & $A_{4}$ & 0.7944 & 0.6163 & $A_{4}$ & 0.4294 \\
$A_{5}$ & 0.7252 & 0.5268 & $A_{5}$ & 0.7180 & 0.5331 & $A_{5}$ & 0.5865 \\
\hline
\end{tabular}

TABLE 15: Degree of relative closeness for each alternative.

\begin{tabular}{llllrr}
\hline$e_{H C}^{1}$ & $N\left(A_{i}\right)$ & $e_{E}$ & $N\left(A_{i}\right)$ & $e_{\min / \max }$ & $A_{1}$ \\
\hline$A_{1}$ & 0.4765 & $A_{1}$ & 0.4688 & $A_{2}$ & 0.4846 \\
$A_{2}$ & 0.6391 & $A_{2}$ & 0.6305 & $A_{3}$ & 0.6432 \\
$A_{3}$ & 0.5601 & $A_{3}$ & 0.5563 & $A_{4}$ & 0.5571 \\
$A_{4}$ & 0.5793 & $A_{4}$ & 0.5631 & $A_{5}$ & 0.5905 \\
$A_{5}$ & 0.5792 & $A_{5}$ & 0.5739 & 0.5843 \\
\hline
\end{tabular}

the closeness degrees are made in an increasing order. Therefore, our analysis shows that the most feasible alternative is $A_{2}$ which is unanimously chosen by all proposed entropy measures.

\section{Conclusions}

In this paper, we have proposed new fuzzy entropy measures for PFSs based on probabilistic-type, distance, Pythagorean 
TABLE 16: Ranking of alternative for different entropies.

\begin{tabular}{llc}
\hline Method & \multicolumn{1}{c}{ Ranking } & Best alternative \\
\hline$e_{H C}^{1}$ & $A_{1} \prec A_{3} \prec A_{5} \prec A_{4} \prec A_{2}$ & $A_{2}$ \\
$e_{E}$ & $A_{1} \prec A_{3} \prec A_{4} \prec A_{5} \prec A_{2}$ & $A_{2}$ \\
$e_{\min / \max }$ & $A_{1} \prec A_{3} \prec A_{5} \prec A_{4} \prec A_{2}$ & $A_{2}$ \\
\hline
\end{tabular}

index, and min-max operator. We have also extended nonprobabilistic entropy to $\sigma$-entropy for PFSs. The entropy measures are considered especially for PFSs on finite universes of discourses. As these are not only used in purposes of computing environment, but also used in more general cases for large universal sets. Structured linguistic variables are used to analyze and compare behaviors and performance of the proposed entropies for PFSs in different Pythagorean fuzzy environments. We have examined and analyzed these comparison results obtained from these entropy measures and then selected appropriate entropies which can be useful and also be helpful to decide fuzziness of PFSs more clearly and efficiently. We have utilized our proposed methods to perform comparison analysis with the most recently developed entropy measure for PFSs. In this connection, we have demonstrated a simple example and a problem involving MCDM to show the advantages of our suggested methods. Finally, the proposed entropy measures of PFSs are applied in an application to multicriterion decision making for ranking China-Pakistan Economic Corridor projects. Based on obtained results, we conclude that the proposed entropy measures for PFSs are reasonable, intuitive, and well suited in handling different kinds of problems, involving linguistic variables and multicriterion decision making in Pythagorean fuzzy environment.

\section{Data Availability}

All data are included in the manuscript.

\section{Conflicts of Interest}

The authors declare that they have no conflicts of interest.

\section{References}

[1] L. A. Zadeh, "Fuzzy sets," Information and Computation, vol. 8, pp. 338-353, 1965.

[2] I. B. Turksen, "Interval valued fuzzy sets based on normal forms," Fuzzy Sets and Systems, vol. 20, no. 2, pp. 191-210, 1986.

[3] J. M. Mendel and R. I. B. John, "Type-2 fuzzy sets made simple," IEEE Transactions on Fuzzy Systems, vol. 10, no. 2, pp. 117-127, 2002.

[4] R. R. Yager, "On the theory of bags," International Journal of General Systems: Methodology, Applications, Education, vol. 13, no. 1, pp. 23-37, 1987.

[5] K. T. Atanassov, "Intuitionistic fuzzy sets," Fuzzy Sets and Systems, vol. 20, no. 1, pp. 87-96, 1986.

[6] V. Torra, "Hesitant fuzzy sets," International Journal of Intelligent Systems, vol. 25, no. 6, pp. 529-539, 2010.
[7] V. Torra and Y. Narukawa, "On hesitant fuzzy sets and decision," in Proceedings of the IEEE International Conference on Fuzzy Systems, pp. 1378-1382, Jeju-do, Republic of Korea, August 2009.

[8] R. R. Yager and A. M. Abbasov, "Pythagorean membership grades, complex numbers, and decision making," International Journal of Intelligent Systems, vol. 28, no. 5, pp. 436-452, 2013.

[9] R. R. Yager, "Pythagorean fuzzy subsets," in Proceedings of the 9th Joint World Congress on Fuzzy Systems and NAFIPS Annual Meeting, IFSA/NAFIPS 2013, pp. 57-61, Edmonton, Canada, June 2013.

[10] R. R. Yager, "Pythagorean membership grades in multicriterion decision making," IEEE Transactions on Fuzzy Systems, vol. 22, no. 4, pp. 958-965, 2014.

[11] X. L. Zhang and Z. S. Xu, "Extension of TOPSIS to multiple criteria decision making with pythagorean fuzzy sets," International Journal of Intelligent Systems, vol. 29, no. 12, pp. 1061-1078, 2014.

[12] X. L. Zhang, "A novel approach based on similarity measure for Pythagorean fuzzymulti-criteria group decision making," International Journal of Intelligence Systems, vol. 31, pp. 593-611, 2016.

[13] P. Ren, Z. Xu, and X. Gou, "Pythagorean fuzzy TODIM approach to multi-criteria decision making," Applied Soft Computing, vol. 42, pp. 246-259, 2016.

[14] X. D. Peng and Y. Yang, "Pythagorean fuzzy Choquet integral based MABAC method for multiple attribute group decision making," International Journal of Intelligent Systems, vol. 31, no. 10, pp. 989-1020, 2016.

[15] X. Zhang, "Multicriteria Pythagorean fuzzy decision analysis: A hierarchical QUALIFLEX approach with the closeness indexbased ranking methods," Information Sciences, vol. 330, pp. 104124, 2016.

[16] X. Peng, H. Yuan, and Y. Yang, "Pythagorean fuzzy information measures and their applications," International Journal of Intelligent Systems, vol. 32, no. 10, pp. 991-1029, 2017.

[17] R. Zhang, J. Wang, X. Zhu, M. Xia, and M. Yu, "Some Generalized Pythagorean Fuzzy Bonferroni Mean Aggregation Operators with Their Application to Multiattribute Group Decision-Making," Complexity, vol. 2017, Article ID 5937376, 16 pages, 2017.

[18] D. Liang and Z. Xu, "The new extension of TOPSIS method for multiple criteria decision making with hesitant Pythagorean fuzzy sets," Applied Soft Computing, vol. 60, pp. 167-179, 2017.

[19] L. Pérez-Domínguez, L. A. Rodríguez-Picón, A. AlvaradoIniesta, D. Luviano Cruz, and Z. Xu, "MOORA under Pythagorean Fuzzy Set for Multiple Criteria Decision Making," Complexity, vol. 2018, Article ID 2602376, 10 pages, 2018.

[20] W. Xue, Z. Xu, X. Zhang, and X. Tian, "Pythagorean fuzzy LINMAP method based on the entropy theory for railway project investment decision making," International Journal of Intelligent Systems, vol. 33, no. 1, pp. 93-125, 2018.

[21] L. Zhang and F. Meng, "An approach to interval-valued hesitant fuzzy multiattribute group decision making based on the generalized Shapley-Choquet integral," Complexity, vol. 2018, Article ID 3941847, 19 pages, 2018.

[22] A. Guleria and R. K. Bajaj, "Pythagorean fuzzy information measure for multicriteria decision making problem," Advances in Fuzzy Systems-Applications and Theory, vol. 2018, Article ID 8023013, 11 pages, 2018.

[23] M. S. Yang and Z. Hussain, "Distance and similarity measures of hesitant fuzzy sets based on Hausdorff metric with applications 
to multi-criteria decision making and clustering," Soft Computing, 2018.

[24] Z. Hussain and M.-S. Yang, "Entropy for hesitant fuzzy sets based on Hausdorff metric with construction of hesitant fuzzy TOPSIS," International Journal of Fuzzy Systems, vol. 20, no. 8, pp. 2517-2533, 2018.

[25] A. de Luca and S. Termini, "A definition of a nonprobabilistic entropy in the setting of fuzzy sets theory," Information and Computation, vol. 20, pp. 301-312, 1972.

[26] R. R. Yager, "On the measure of fuzziness and negation. Part I: membership in the unit interval," International Journal of General Systems, vol. 5, no. 4, pp. 189-200, 1979.

[27] B. Kosko, "Fuzzy entropy and conditioning," Information Sciences, vol. 40, no. 2, pp. 165-174, 1986.

[28] X. C. Liu, "Entropy, distance measure and similarity measure of fuzzy sets and their relations," Fuzzy Sets and Systems, vol. 52, no. 3, pp. 305-318, 1992.

[29] N. R. Pal and S. K. Pal, "Some properties of the exponential entropy," Information Sciences, vol. 66, no. 1-2, pp. 119-137, 1992.

[30] J.-L. Fan and Y.-L. Ma, "Some new fuzzy entropy formulas," Fuzzy Sets and Systems, vol. 128, no. 2, pp. 277-284, 2002.

[31] P. Burillo and H. Bustince, "Entropy on intuitionistic fuzzy sets and on interval-valued fuzzy sets," Fuzzy Sets and Systems, vol. 78, no. 3, pp. 305-316, 1996.

[32] E. Szmidt and J. Kacprzyk, "Entropy for intuitionistic fuzzy sets," Fuzzy Sets and Systems, vol. 118, no. 3, pp. 467-477, 2001.

[33] E. Szmidt and J. Baldwin, "Entropy for intuitionistic fuzzy set theory and mass assignment theory," Notes on IFSs, vol. 10, pp. 15-28, 2004.

[34] W. L. Hung and M. S. Yang, "Fuzzy entropy on intuitionistic fuzzy sets," International Journal of Intelligent Systems, vol. 21, no. 4, pp. 443-451, 2006.

[35] J. Havrda and F. S. Charvát, "Quantification method of classification processes. Concept of structural $\alpha$-entropy," Kybernetika, vol. 3, pp. 30-35, 1967. 


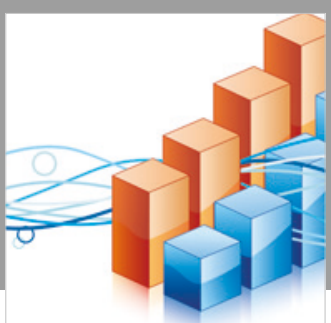

Advances in

Operations Research

\section{-n-m}
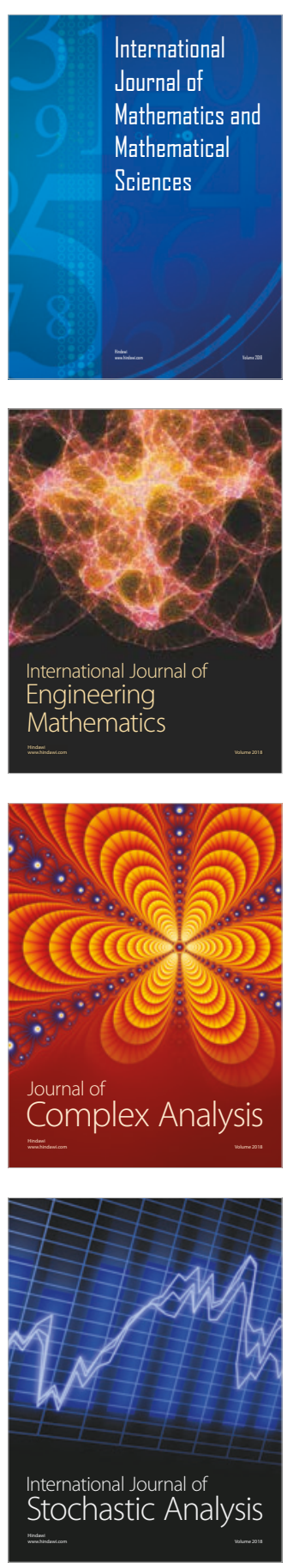
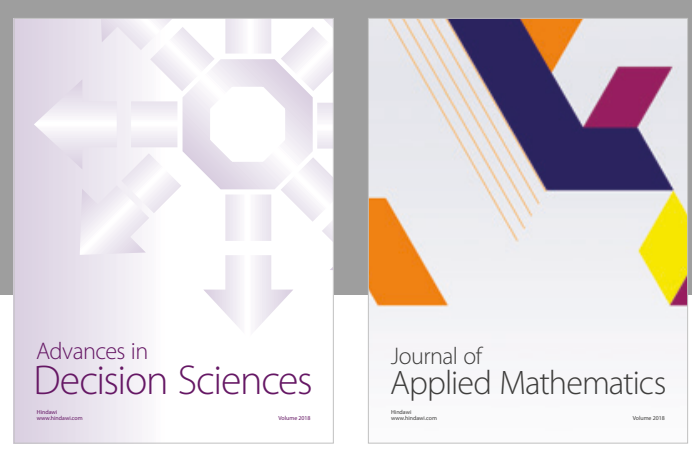

Journal of

Applied Mathematics
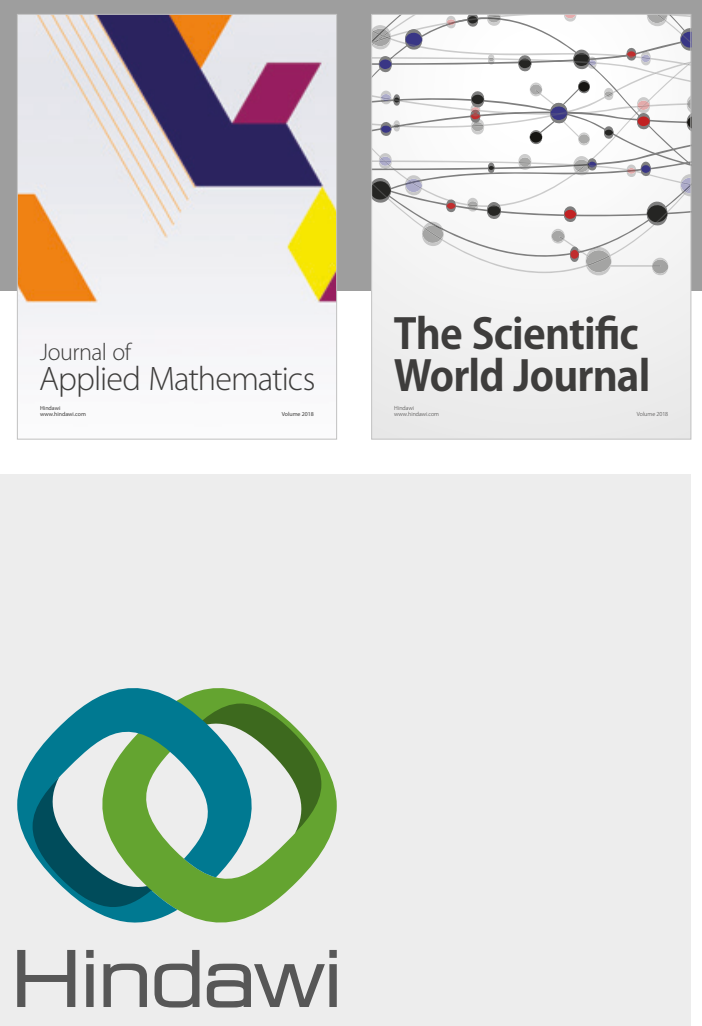

Submit your manuscripts at

www.hindawi.com

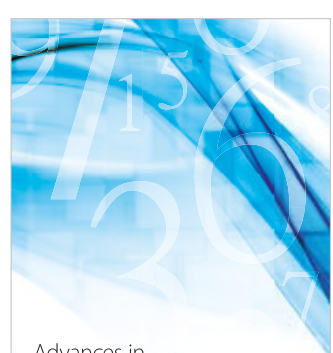

Advances in
Numerical Analysis
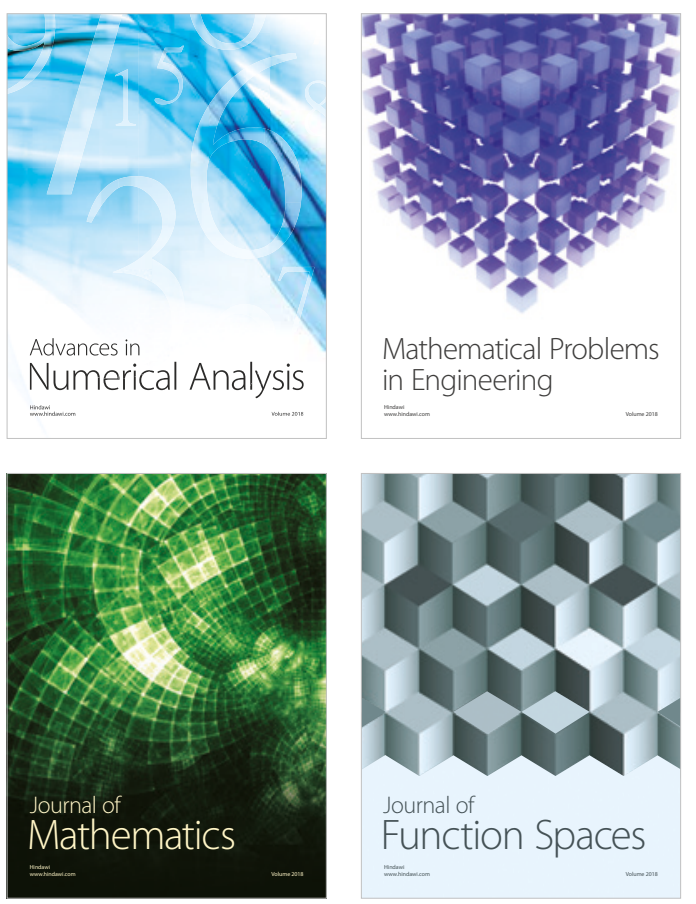

Mathematical Problems in Engineering

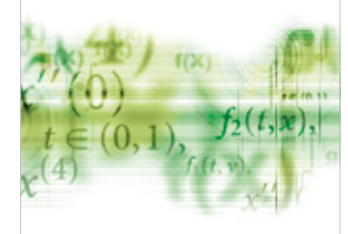

International Journal of

Differential Equations

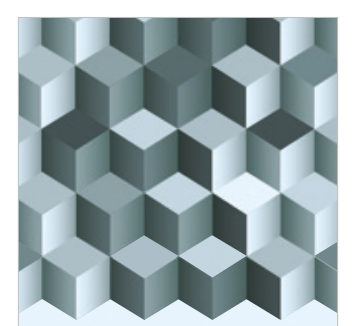

Journal of

Function Spaces
The Scientific

World Journal

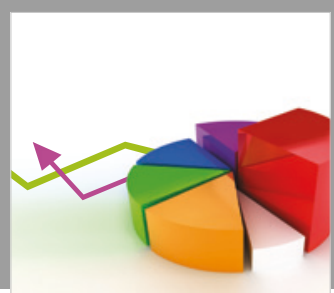

Journal of

Probability and Statistics
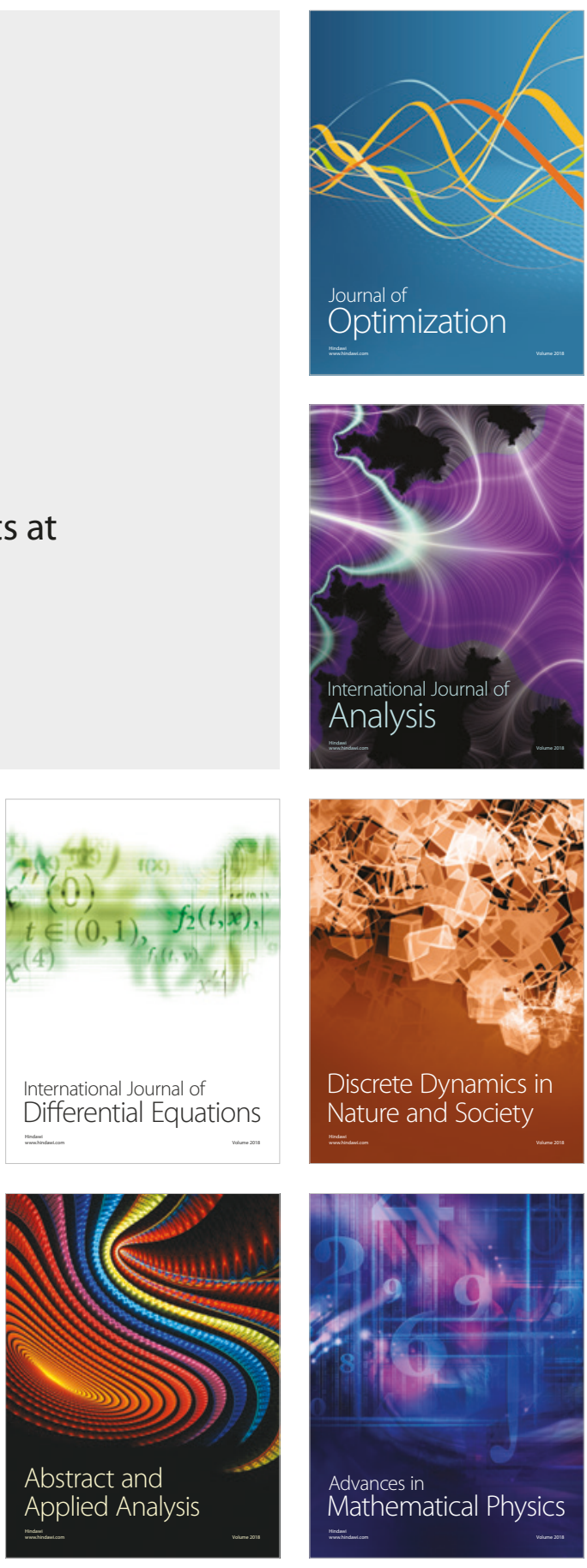